\title{
Supplementary Material - A Bayesian Machine Learning Approach to the Quantification of Uncertainties on Ab Initio Potential Energy Surfaces
}

\author{
S. Venturi and M. Panesi* \\ University of Illinois at Urbana-Champaign, Urbana, Illinois 61801, USA \\ R. L. Jaffe \\ NASA Ames Research Center, Moffett Field, CA 94035-1000 \\ (Dated: May 26, 2020)
}

*mpanesi@illinois.edu 


\section{CONTENTS}

I. Convergence of QCT and CG-QCT Calculations $\quad$ S3

$\begin{array}{ll}\text { A. QCT + ME Calculations } & \text { S4 }\end{array}$

B. CG-QCT200 + CGM200 Calculations $\quad$ S6

$\begin{array}{ll}\text { II. Verification Test Cases for the PIP-BNN } & \text { S8 }\end{array}$

$\begin{array}{ll}\text { A. Errors at Data Points } & \text { S9 }\end{array}$

B. Gas QoIs at $10000 \mathrm{~K} \quad$ S12

III. Further Results for the PIP-BNN applied to $1^{1} A^{\prime}$ and $2^{5} A^{\prime}$ PESs $\quad$ S15

$\begin{array}{ll}\text { A. Errors at Data Points } & \text { S15 }\end{array}$

$\begin{array}{ll}\text { B. PESs 2D Cuts } & \text { S17 }\end{array}$

$\begin{array}{lr}\text { C. PESs 3D Cuts } & \text { S18 }\end{array}$

$\begin{array}{ll}\text { 1. Results for } 1^{1} A^{\prime} \text { PESs } & \text { S18 }\end{array}$

2. Results for $2^{5} A^{\prime}$ PESs $\quad$ S19

$\begin{array}{ll}\text { D. Rate Coefficients at } \mathrm{T}=10000 \mathrm{~K} & \mathrm{~S} 22\end{array}$

1. Results for $2^{5} A^{\prime}$ PESs $\quad$ S22

E. Gas Quantities of Interest at $\mathrm{T}=10000 \mathrm{~K} \quad \mathrm{~S} 24$

$\begin{array}{ll}\text { 1. Results for } 2^{5} A^{\prime} \text { PESs } & \text { S24 }\end{array}$

$\begin{array}{ll}\text { F. Gas Quantities of Interest at } \mathrm{T}=2500 \mathrm{~K} & \text { S28 }\end{array}$

$\begin{array}{ll}\text { 1. Results for } 1^{1} A^{\prime} \text { PESs } & \text { S28 }\end{array}$

2. Results for $2^{5} A^{\prime}$ PESs $\quad$ S29

G. Gas Quantities as Functions of Temperature $\quad$ S30

H. Gas QoI Sensitivities to the $2^{5} A^{\prime}$ PES Uncertainties $\quad$ S33

1. Vibrational Relaxation Times $\quad$ S33

2. Vibrational Relaxation Times Excluding Exchange Processes $\quad$ S34

3. Rotational Relaxation Times $\quad$ S35

4. Global Dissociation Rates at Equilibrium $\quad$ S36

5. Global Dissociation Rates at QSS $\quad$ S37

6. Global Exchange Rates at Equilibrium $\quad$ S38

$\begin{array}{ll}\text { 7. Mole Fractions } & \text { S39 }\end{array}$ 


\section{CONVERGENCE OF QCT AND CG-QCT CALCULATIONS}

In this section, the convergences of the QCT and CG-QCT calculations are presented, with the objective of showing that a further increase in the number of computed trajectories would not significantly change the predicted values of the gas QoIs.

Four test cases are analyzed, all of them involving the simulations of gas mixtures composed by atomic and molecular oxygen undergoing thermal baths in an isochoric reactor. The interactions between $\mathrm{O}_{2}$ and $\mathrm{O}$ are assumed to take place only on the first singlet surface, $1^{1} A^{\prime}$. We preferred $1^{1} A^{\prime}$ to the other $8 \mathrm{O}_{2}+\mathrm{O}$ available PESs due to the relatively large number of resulting processes that its low exchange barrier makes likely to happen.

The gas is considered in initial thermal equilibrium at $T_{\text {Tran }}^{0}=T_{\text {Int }}^{0}=1400 \mathrm{~K}$, pressure $P=9940$ $\mathrm{Pa}$, and mole fraction composition of $5 \%$ of $\mathrm{O}$ and $95 \%$ of $\mathrm{O}_{2}$. At the first instants of the simulations, the translational temperature is artificially raised to higher $T_{\operatorname{Tran}}\left(T_{\operatorname{Tran}}=2500 \mathrm{~K}\right.$ for the first and the third test cases, and $T_{\text {Tran }}=10000 \mathrm{~K}$ for the second and the fourth ones), and then kept constant for all the following times. The heat baths are simulated by means of ME (test cases 1 and 2) and 200 bins CGM (test cases 3 and 4).

In the first two test cases, the results obtained by using StS rates computed through 20000 trajectories per level are compared to the ones produced by StS rates generated through 100000 trajectories per level. In the remaining two cases, instead, the results of CGM obtained by using bin rates grouped from the $\mathrm{StS}$ ones are compared to the outcomes of CGM with bin rates directly computed through CG-QCT, with 50000 trajectories per group. 


\section{A. QCT + ME Calculations}

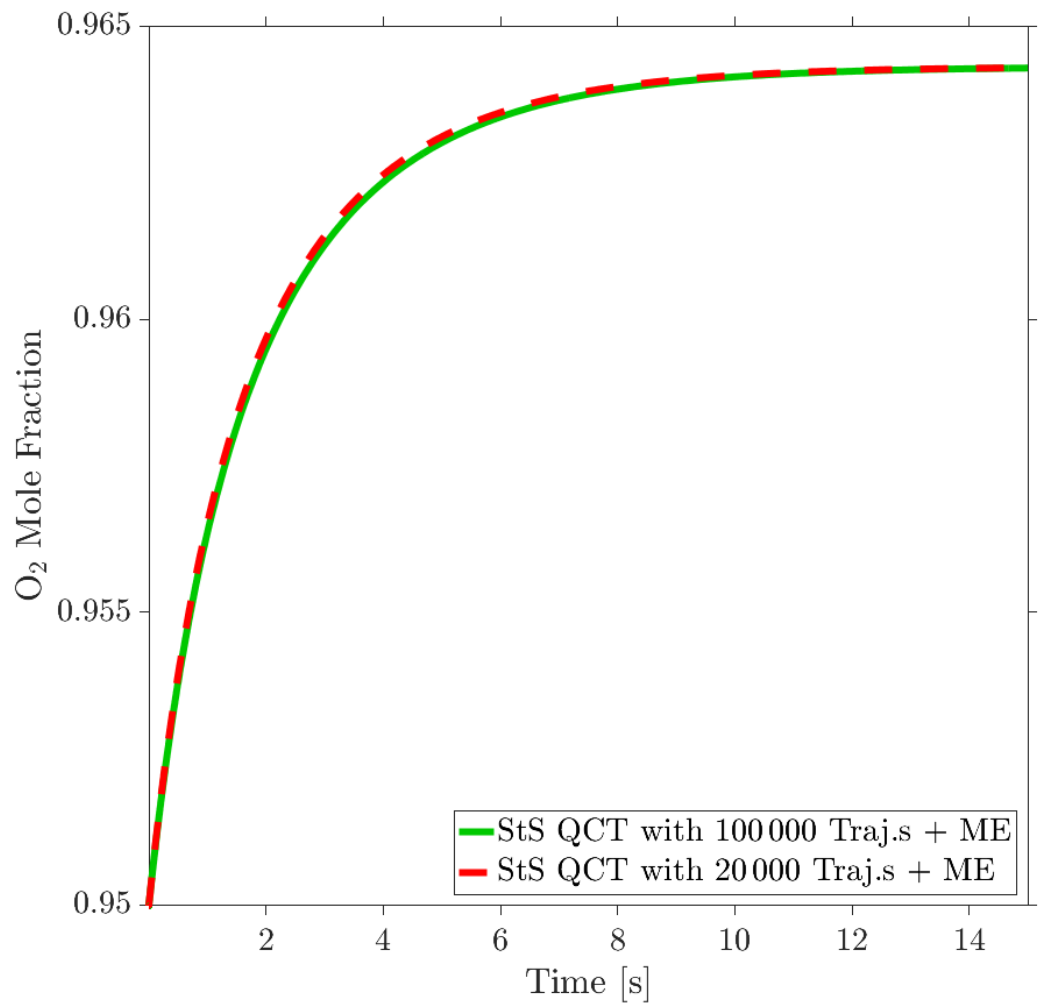

FIG. S1: Evolution of $\mathrm{O}_{2}$ mole fraction in a StS heat bath at constant $T_{\text {Tran }}=2500 \mathrm{~K}$. The rates for the ME calculations are computed from 100000 (green solid line) and 20000 (red dashed line) trajectories per level.

TABLE S1: Gas QoIs at $T_{\text {Tran }}=2500 \mathrm{~K}$ from StS Calculations

\begin{tabular}{|l|c|c|}
\hline \hline QoI & 20000 Traj.s & 100000 Traj.s \\
\hline \hline$p \tau_{V}[a t m * s]$ & $2.308 \mathrm{e}-09$ & $2.315 \mathrm{e}-09$ \\
$p \tau_{R}[a t m * s]$ & $2.116 \mathrm{e}-09$ & $2.136 \mathrm{e}-09$ \\
$k_{Q S S}^{D}\left[\mathrm{~cm}^{3} / \mathrm{s}\right]$ & - & - \\
$k_{E q}^{D}\left[\mathrm{~cm}^{3} / \mathrm{s}\right]$ & $3.077 \mathrm{e}-19$ & $2.995 \mathrm{e}-19$ \\
$k_{Q S S}^{E}\left[\mathrm{~cm}^{3} / \mathrm{s}\right]$ & - & - \\
$k_{E q}^{E}\left[\mathrm{~cm}^{3} / \mathrm{s}\right]$ & $1.257 \mathrm{e}-10$ & $1.266 \mathrm{e}-10$ \\
\hline
\end{tabular}




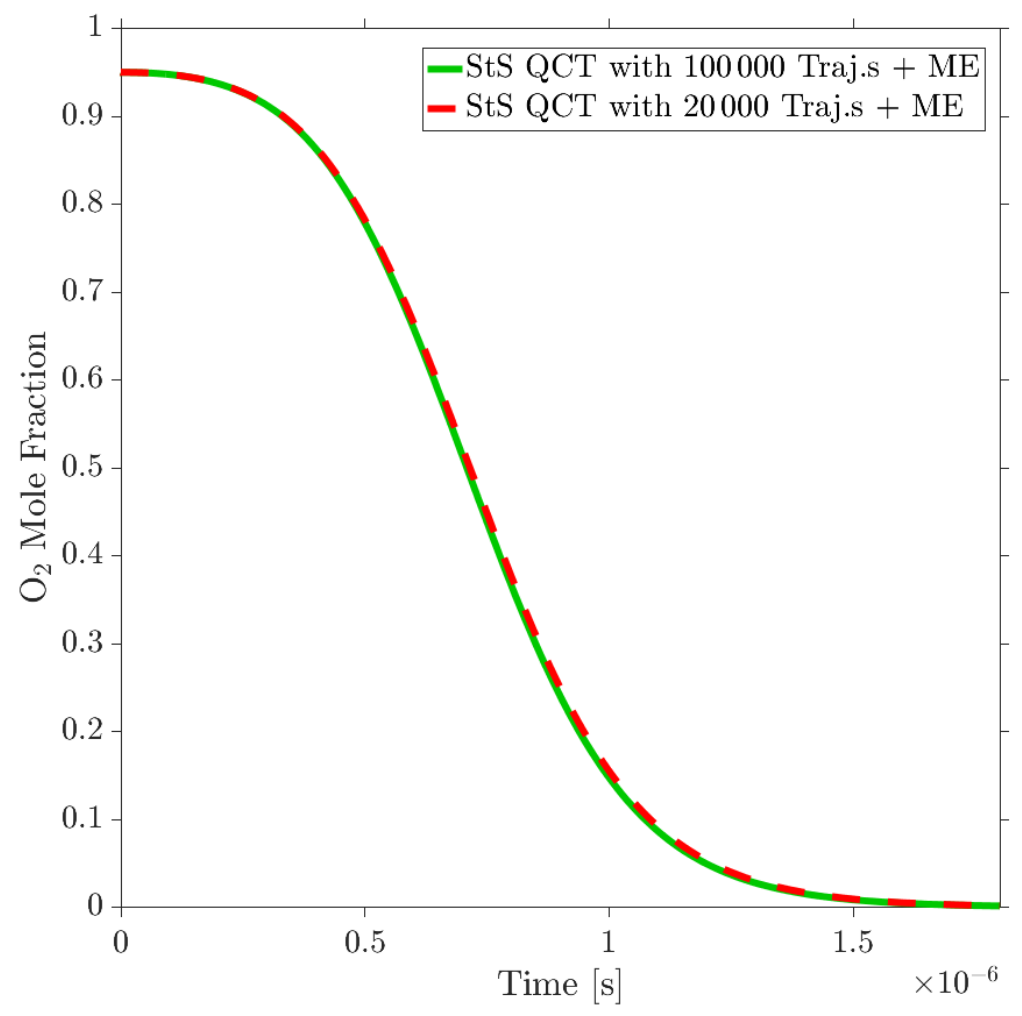

FIG. S2: Evolution of $\mathrm{O}_{2}$ mole fraction in a StS heat bath at constant $T_{T r a n}=10000 \mathrm{~K}$. The rates for the ME calculations are computed from 100000 (green solid line) and 20000 (red dashed line) trajectories per level.

TABLE S2: Gas QoIs at $T_{\text {Tran }}=10000 \mathrm{~K}$ from StS Calculations

\begin{tabular}{|l|c|c|}
\hline \hline QoI & 20000 Traj.s & 100000 Traj.s \\
\hline \hline$p \tau_{V}[a t m * s]$ & $7.208 \mathrm{e}-09$ & $7.205 \mathrm{e}-09$ \\
$p \tau_{R}[a t m * s]$ & $5.454 \mathrm{e}-09$ & $5.459 \mathrm{e}-09$ \\
$k_{Q S S}^{D}\left[\mathrm{~cm}^{3} / \mathrm{s}\right]$ & $5.932 \mathrm{e}-12$ & $6.011 \mathrm{e}-12$ \\
$k_{E q}^{D}\left[\mathrm{~cm}^{3} / \mathrm{s}\right]$ & $9.570 \mathrm{e}-12$ & $9.654 \mathrm{e}-12$ \\
$k_{Q S S}^{E}\left[\mathrm{~cm}^{3} / \mathrm{s}\right]$ & $3.128 \mathrm{e}-10$ & $3.116 \mathrm{e}-10$ \\
$k_{E q}^{E}\left[\mathrm{~cm}^{3} / \mathrm{s}\right]$ & $3.201 \mathrm{e}-10$ & $3.190 \mathrm{e}-10$ \\
\hline
\end{tabular}




\section{B. CG-QCT200 + CGM200 Calculations}

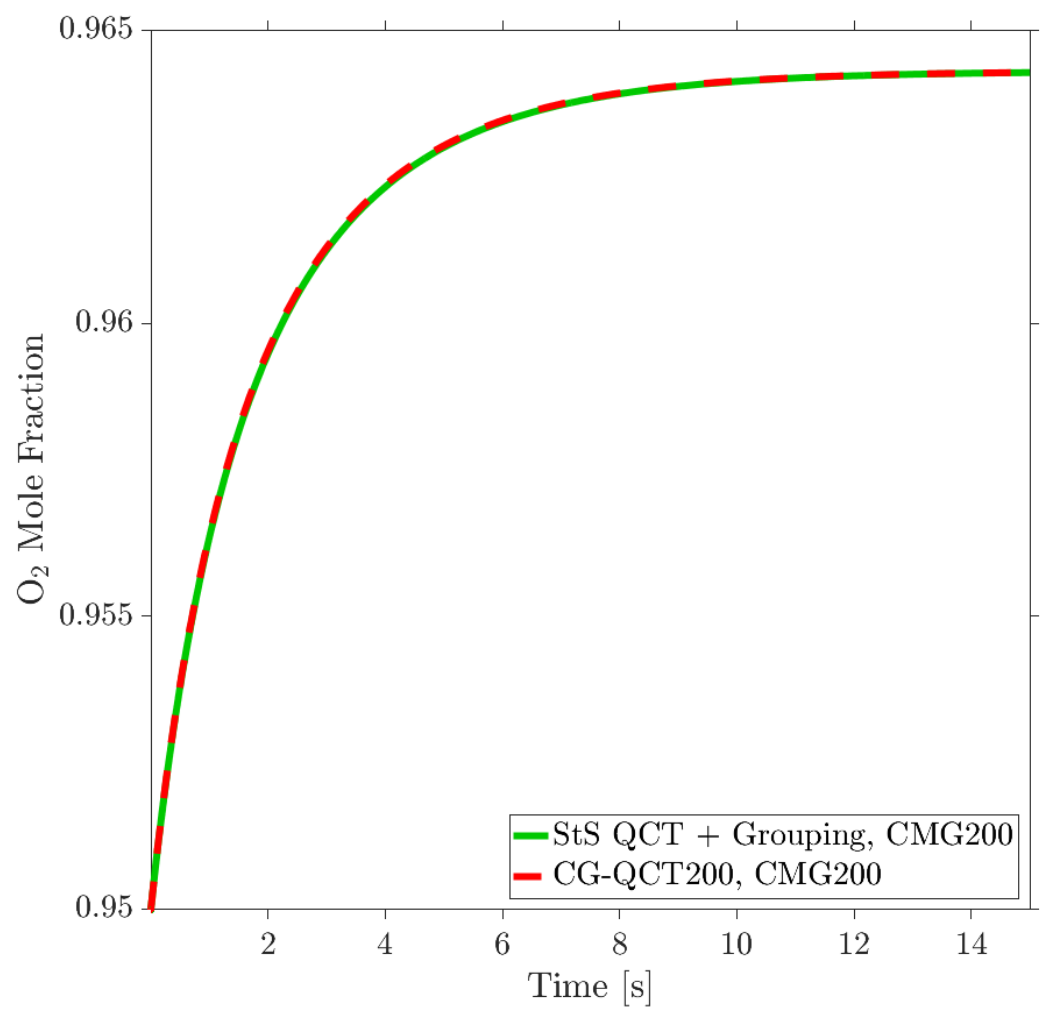

FIG. S3: Evolution of $\mathrm{O}_{2}$ mole fraction in a heat bath at constant $T_{\text {Tran }}=2500 \mathrm{~K}$ simulated by means of CGM200. The results are obtained through bin rates computed by grouping the StS ones (green solid line) or directly by CG-QCT calculations (red dashed line).

TABLE S3: Gas QoIs at $T_{\text {Tran }}=2500 \mathrm{~K}$ from CGM200

\begin{tabular}{|l|c|c|}
\hline \hline QoI & CG-QCT200 & QCT + 200 Bins Grouping \\
\hline \hline$p \tau_{V}[a t m * s]$ & $2.039 \mathrm{e}-09$ & $2.035 \mathrm{e}-09$ \\
$p \tau_{R}[a t m * s]$ & $1.543 \mathrm{e}-09$ & $1.540 \mathrm{e}-09$ \\
$k_{Q S S}^{D}\left[\mathrm{~cm}^{3} / \mathrm{s}\right]$ & - & - \\
$k_{E q}^{D}\left[\mathrm{~cm}^{3} / \mathrm{s}\right]$ & $3.009-19$ & $2.977 \mathrm{e}-19$ \\
$k_{Q S S}^{E}\left[\mathrm{~cm}^{3} / \mathrm{s}\right]$ & - & - \\
$k_{E q}^{E}\left[\mathrm{~cm}^{3} / \mathrm{s}\right]$ & $1.275 \mathrm{e}-10$ & $1.266 \mathrm{e}-10$ \\
\hline
\end{tabular}




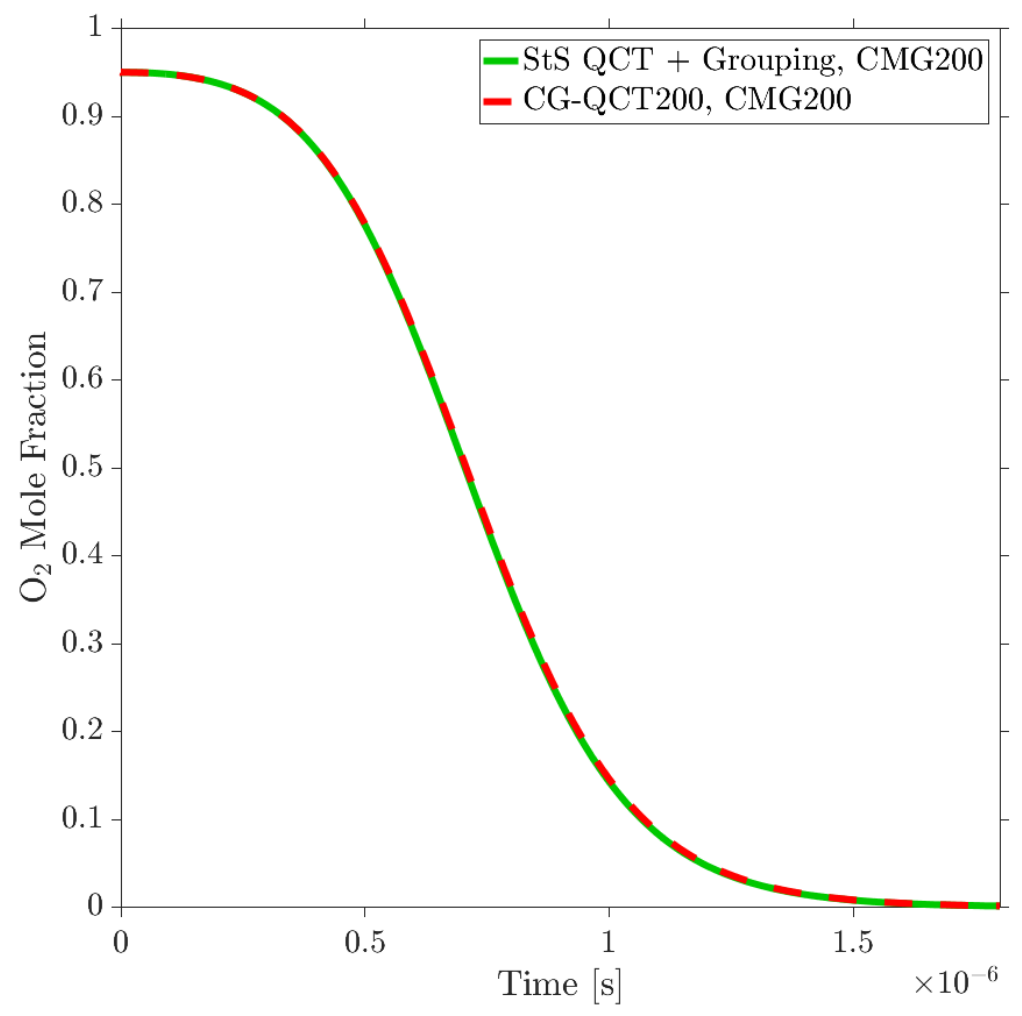

FIG. S4: Evolution of $\mathrm{O}_{2}$ mole fraction in a heat bath at constant $T_{T r a n}=10000 \mathrm{~K}$ simulated by means of CGM200. The results are obtained through bin rates computed by grouping the StS ones (green solid line) or directly by CG-QCT calculations (red dashed line).

TABLE S4: Gas QoIs at $T_{\text {Tran }}=10000 \mathrm{~K}$ from CGM200

\begin{tabular}{|l|c|c|}
\hline \hline QoI & CG-QCT200 & QCT + 200 Bins Grouping \\
\hline$p \tau_{V}[a t m * s]$ & $6.860 \mathrm{e}-09$ & $6.884 \mathrm{e}-09$ \\
$p \tau_{R}[a t m * s]$ & $4.937 \mathrm{e}-09$ & $4.917 \mathrm{e}-09$ \\
$k_{Q S S}^{D}\left[\mathrm{~cm}^{3} / \mathrm{s}\right]$ & $6.020 \mathrm{e}-12$ & $6.054 \mathrm{e}-12$ \\
$k_{E q}^{D}\left[\mathrm{~cm}^{3} / \mathrm{s}\right]$ & $9.651 \mathrm{e}-12$ & $9.654 \mathrm{e}-12$ \\
$k_{Q S S}^{E}\left[\mathrm{~cm}^{3} / \mathrm{s}\right]$ & $3.140 \mathrm{e}-10$ & $3.118 \mathrm{e}-10$ \\
$k_{E q}^{E}\left[\mathrm{~cm}^{3} / \mathrm{s}\right]$ & $3.213 \mathrm{e}-10$ & $3.190 \mathrm{e}-10$ \\
\hline
\end{tabular}




\section{VERIFICATION TEST CASES FOR THE PIP-BNN}

In this section, the PIP-BNN methodology for constructing stochastic PESs is verified. Three different cases are proposed, and in each of them a stochastic PES is generated based on fabricated data. In particular, the geometric arrangements for the data points are selected as follows:

- Test Case 1: The 1617 atomic configurations for the $2^{5} A^{\prime}$ surface reported in Varga et al.'s paper, plus 8383 geometries for which $r_{1}, r_{3}$ and $\alpha$ are sampled through LHS with

$1.5 a_{0}<r_{1}<10.0 a_{0}, 1.5 a_{0}<r_{3}<10.0 a_{0}$, and $35^{\circ}<\alpha<175^{\circ}\left(r_{1}=\overline{O_{A} O_{B}}, r_{3}=\overline{O_{B} O_{C}}\right.$ and $\alpha=\angle O_{A} O_{B} O_{C}$;

- Test Case 2: The 1617 atomic configurations for the $2^{5} A^{\prime}$ surface reported in Varga et al.'s paper, plus 3383 geometries sampled through the same strategy as in Test Case 1;

- Test Case 3: The 1617 atomic configurations for the $2^{5} A^{\prime}$ surface reported in Varga et al.'s paper.

In order to generate labeled data, the corresponding potential energies are obtained by evaluating Varga et al.'s fit for $2^{5} A^{\prime}$ at such geometric arrangements. The results from the three test cases are compared to each other, in order to show that the error in reconstructing Varga et al.'s PES monotonically reduces with increasing the amount of the data points.

The PIP-BNN stochastic PES generated through test case 3 is then adopted for QCT and CGQCT calculations at $T_{\text {Tran }}=10000 \mathrm{~K}$; the resultant StS and group rates are used for simulating a heat bath, with the goal of showing that the gas QoIs predicted by the so constructed PIP-BNN are in well agreement with the ones generated with Varga et al.'s fit. The gas is considered in initial thermal equilibrium at $T_{\text {Tran }}^{0}=T_{\text {Int }}^{0}=1400 \mathrm{~K}$, pressure $P=9940 \mathrm{~Pa}$, and mole fraction composition of $5 \%$ of $\mathrm{O}$ and $95 \%$ of $\mathrm{O}_{2}$. At the first instants of the simulations, the translational temperature is artificially raised to $T_{\operatorname{Tran}}=10000 \mathrm{~K}$, and then kept constant for all the following times. Mole fractions, QSS and equilibrium dissociation rate coefficients, and vibrational and rotational relaxation times are analyzed as quantities of interest. 


\section{A. Errors at Data Points}

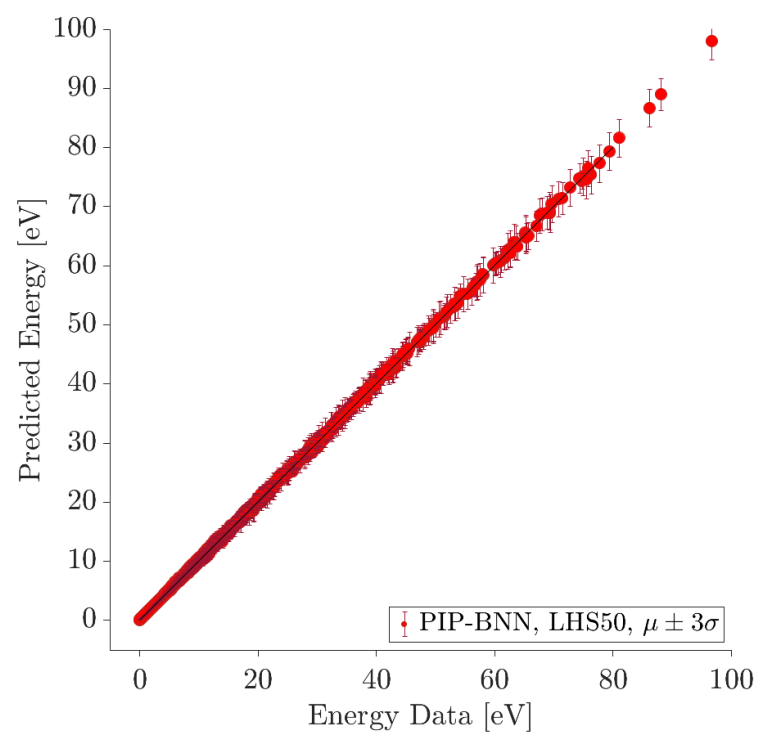

(a) Test Case 1

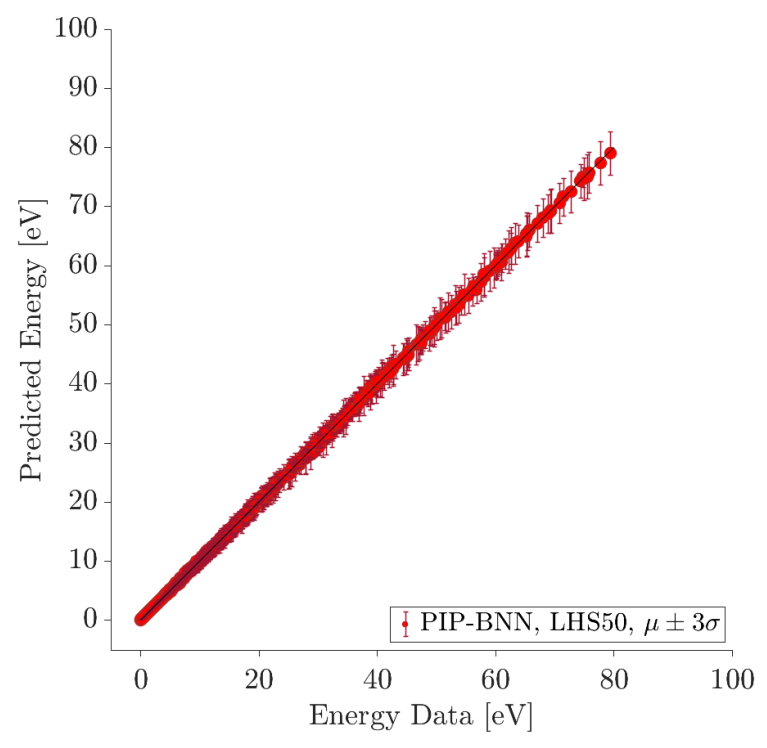

(b) Test Case 2

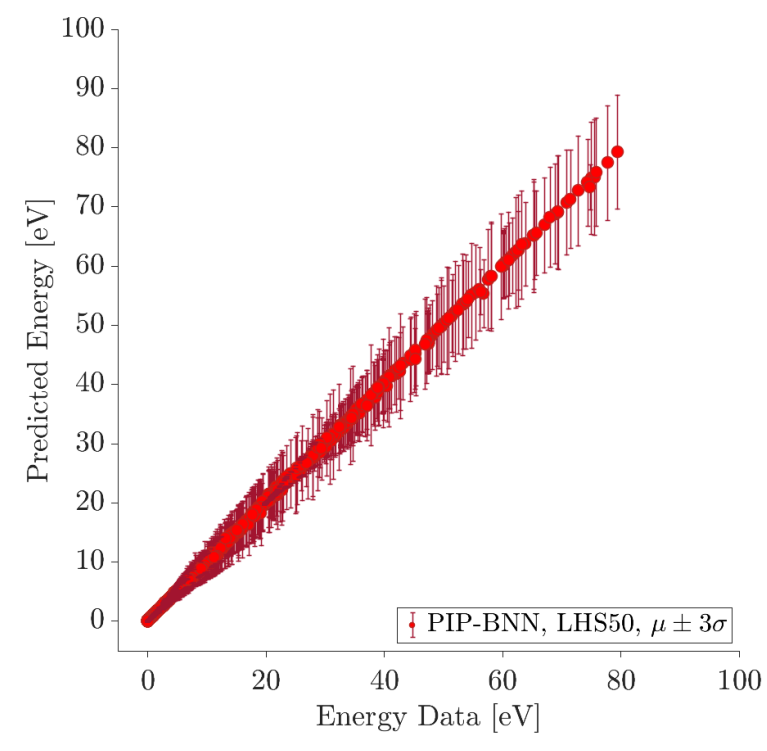

(c) Test Case 3

FIG. S5: Means (red dots) and three-sigma confidence intervals (red lines) for the potential energies computed at the data points using 50 PIP-BNN samples. 


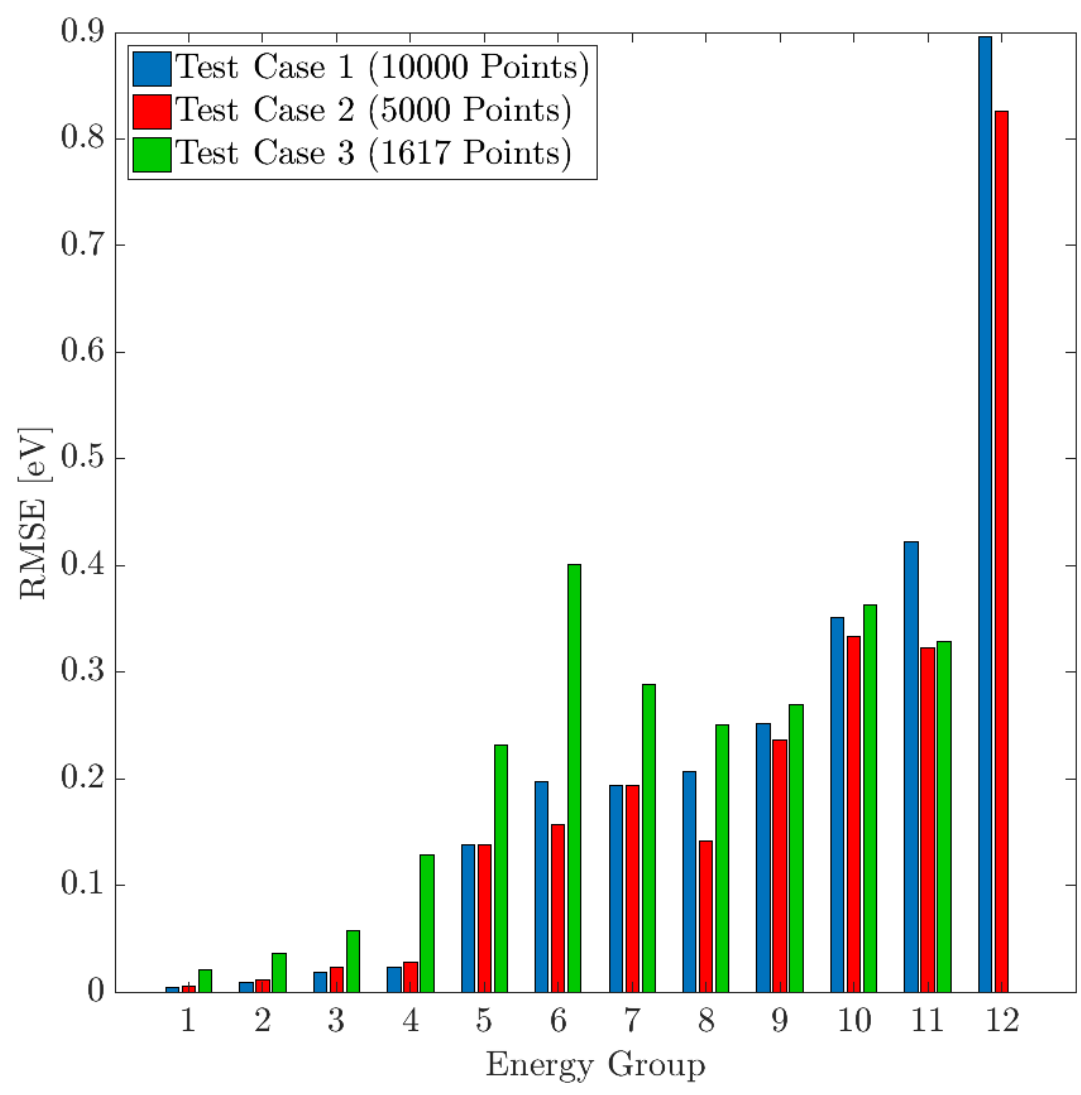

FIG. S6: RMSEs from the mean of the 50 PIP-BNN samples at the data points of $2^{5} A^{\prime}$ for the 3 test cases (blue for case 1, red for case 2 and green for case 3). Based on their potential energies, the data points have been divided in 12 groups, with upper bounds given by

$\boldsymbol{V}_{\text {Max }}=\{2.0 ; 4.0 ; 6.0 ; 8.0 ; 10.0 ; 15.0 ; 20.0 ; 25.0 ; 30.0 ; 50.0 ; 100.0 ; 1000.0\} \mathrm{eV}$ 


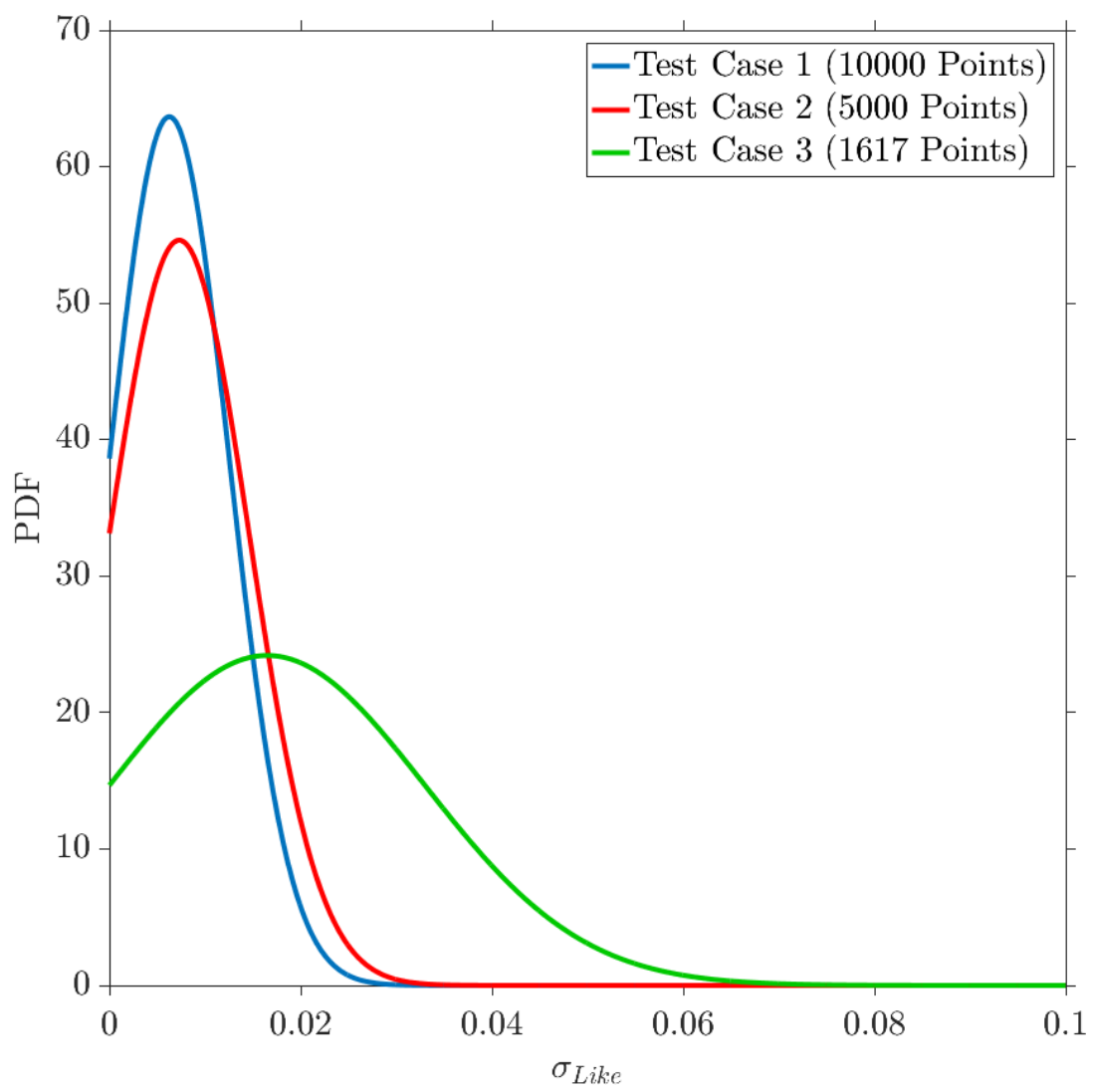

FIG. S7: Posterior distributions of the Likelihood standard deviation for the 3 test cases (blue for case 1, red for case 2 and green for case 3). 


\section{B. Gas QoIs at $10000 \mathrm{~K}$}

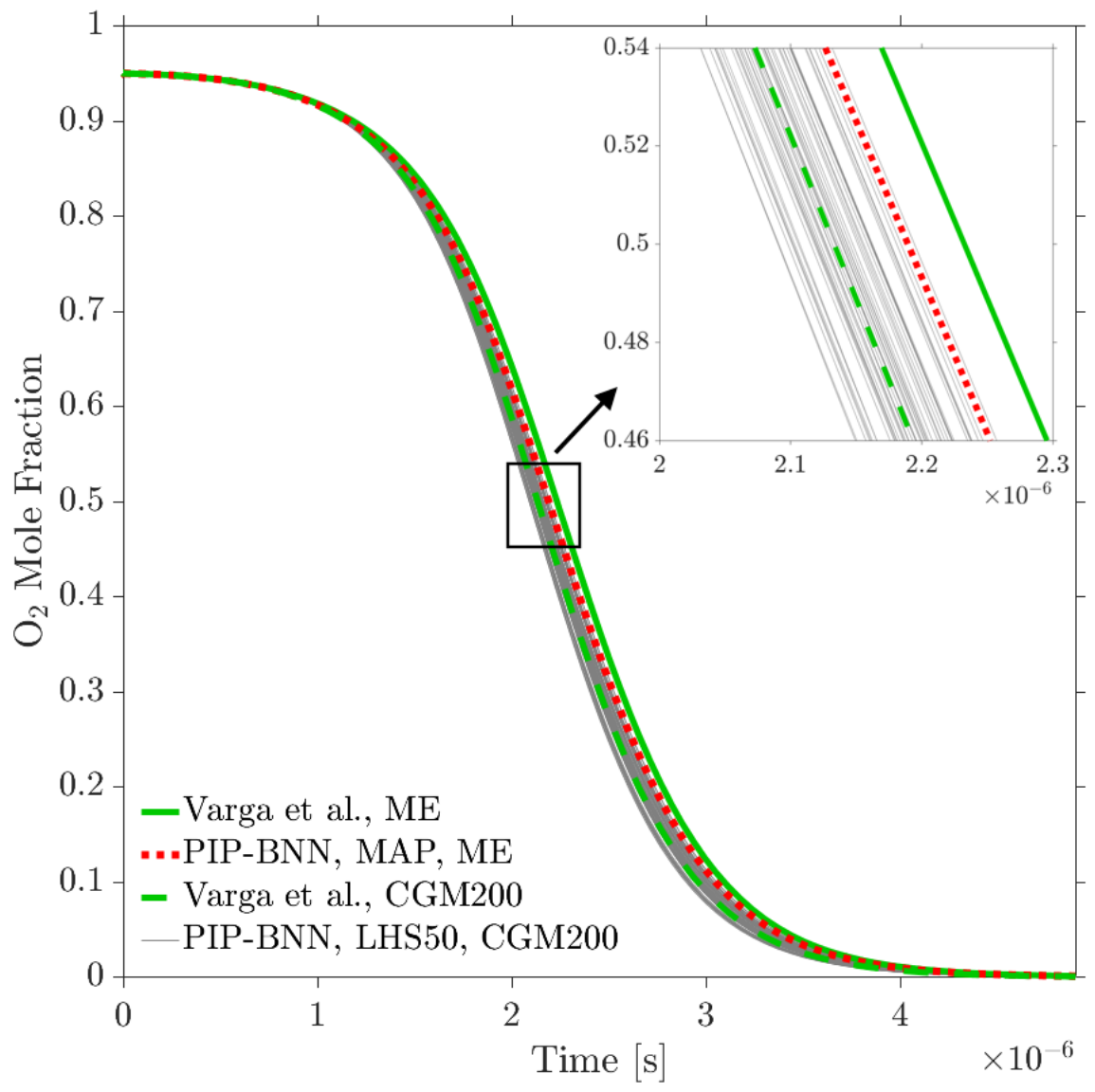

FIG. S8: Evolution of $\mathrm{O}_{2}$ mole fraction, as predicted by ME (green solid line) and CGM200 (green dashed line) using Varga et al.'s fit, by ME using the PIP-BNN MAP surface (red dotted line), and by CGM200 using the 50 PIP-BNN samples (grey narrow lines). The PIP-BNN adopted is the one constructed for test case 3 , and the simulations are performed at $T_{\text {Tran }}=10000 \mathrm{~K}$. 


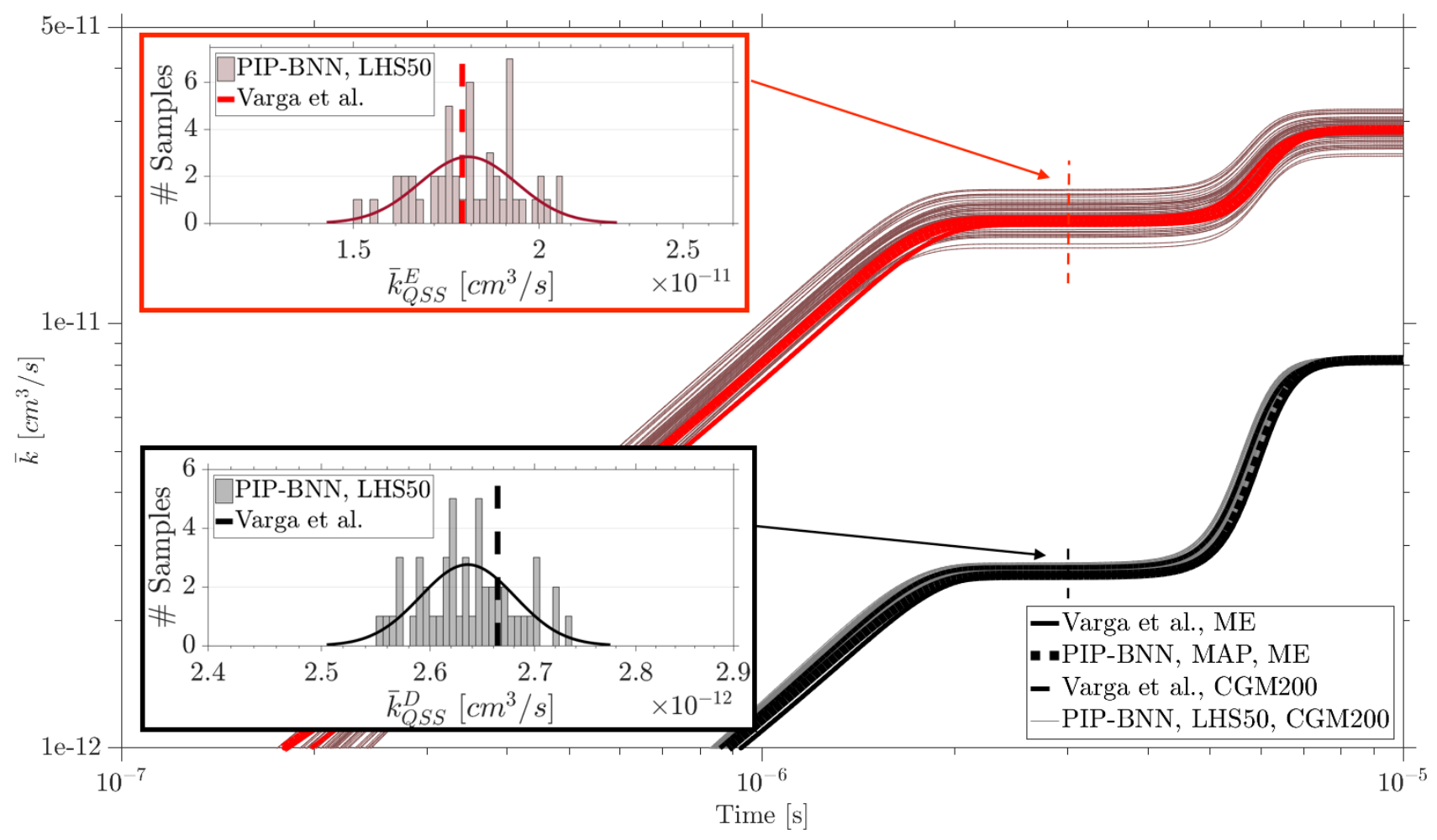

FIG. S9: Evolution of $\mathrm{O}_{2}$ dissociation (lower black part) and exchange (upper red part) averaged rates, as predicted by ME (solid lines) and CGM200 (dashed lines) using Varga et al.'s fit, by ME using the PIP-BNN MAP surface (dotted lines), and by CGM200 using the 50 PIP-BNN samples (narrow lines).

The histograms on the left report the dissociation (lower Subfig.) and exchange (upper Subfig.) QSS rates predicted through such samples, and they compare them to the outcomes of CGM200 using Varga et al.'s fit. The PIP-BNN adopted is the one constructed for test case 3, and the simulations are performed at $T_{\text {Tran }}=10000 \mathrm{~K}$. 


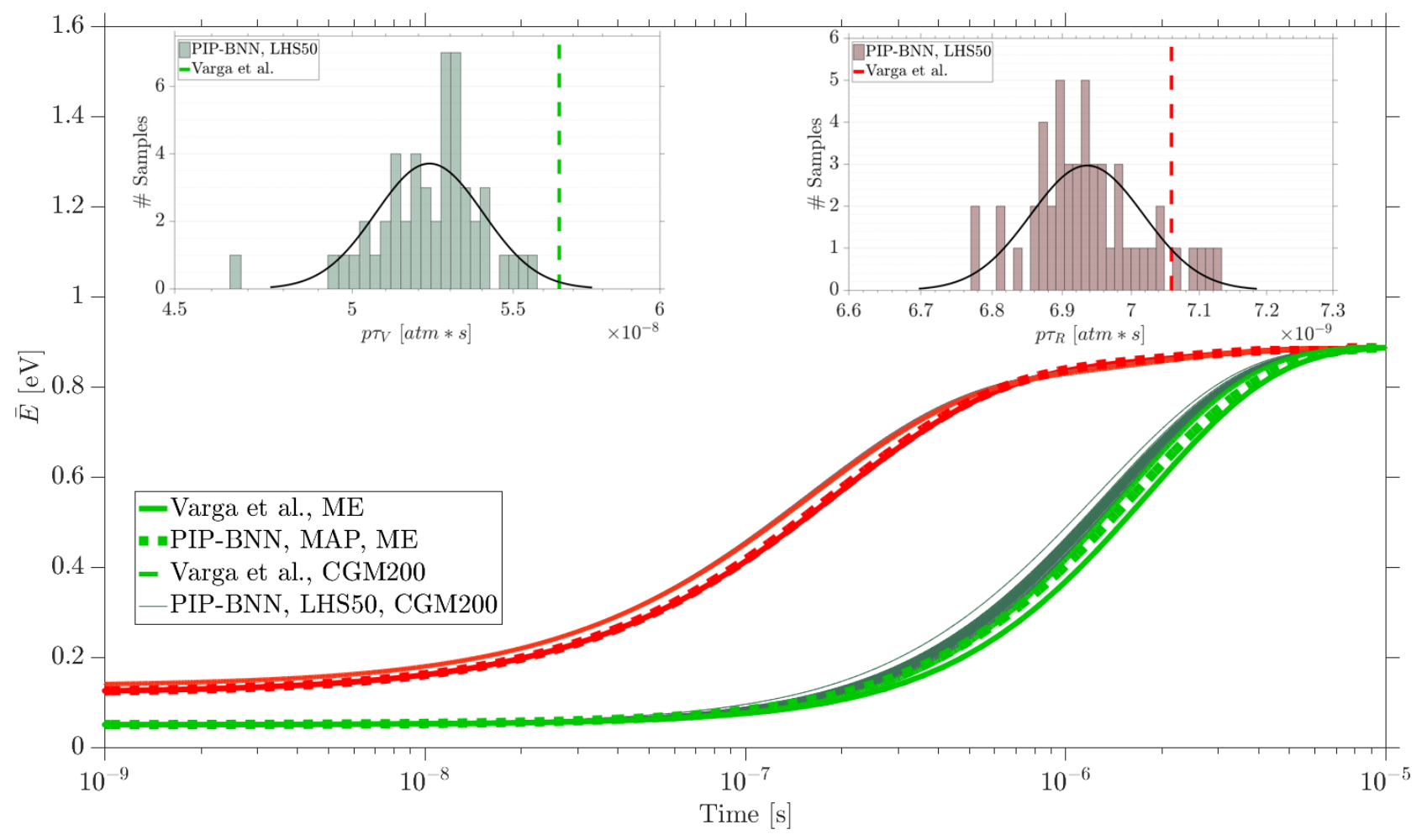

FIG. S10: Evolution of $\mathrm{O}_{2}$ averaged vibrational (green lines) and rotational (red lines) energy, as predicted by ME (solid lines) and CGM200 (dashed lines) using Varga et al.'s fit, by ME using the PIP-BNN MAP surface (dotted lines), and by CGM200 using the 50 PIP-BNN MAP samples (narrow lines). The sub-figures on the top report the histogram of the vibrational and rotational relaxation times predicted through such samples, and they compare them to the outcomes of CGM200 using Varga et al.'s fit. The PIP-BNN adopted is the one constructed for test case 3, and the simulations are performed at $T_{\text {Tran }}=10000 \mathrm{~K}$. 


\section{FURTHER RESULTS FOR THE PIP-BNN APPLIED TO $1^{1} A^{\prime}$ AND $2^{5} A^{\prime}$ PESS}

A. Errors at Data Points

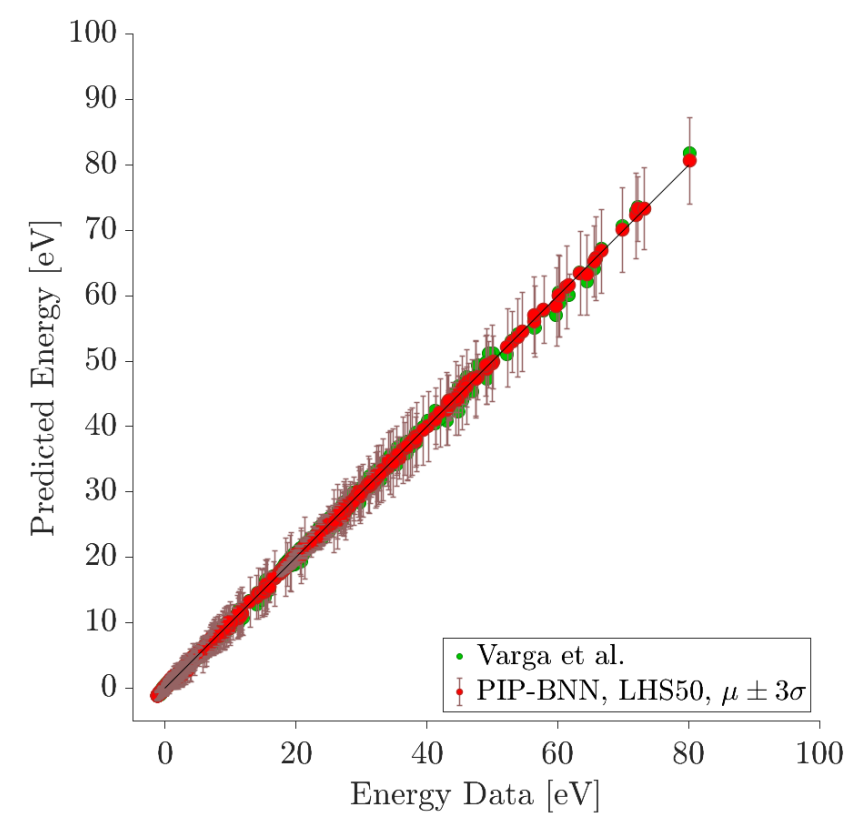

(a)

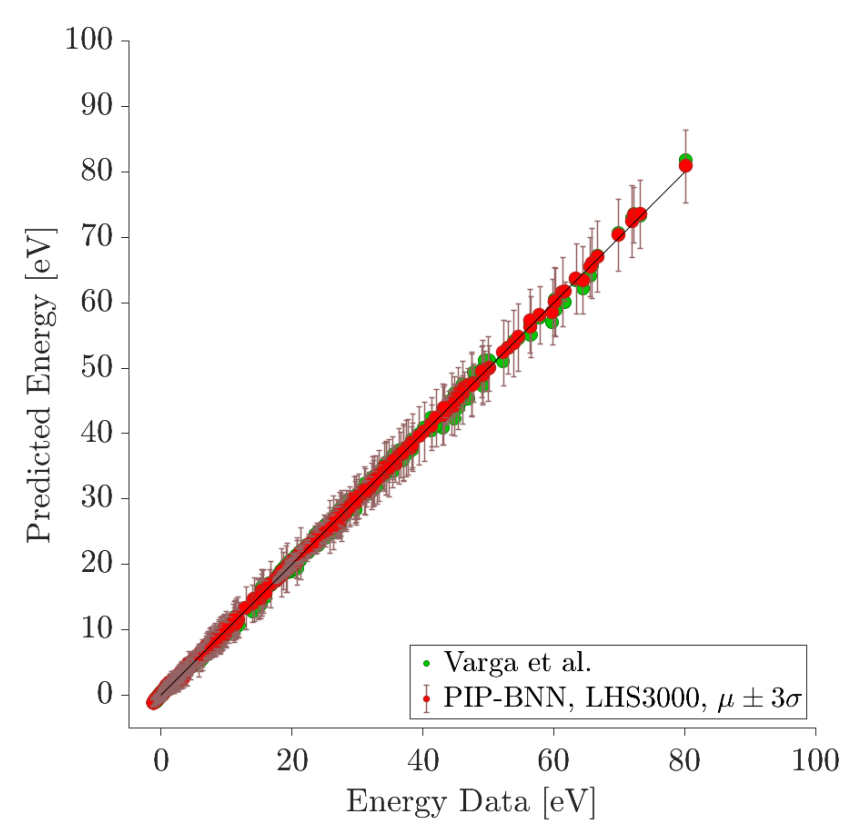

(b)

FIG. S11: Means and three-sigma confidence intervals of the $1^{1} A^{\prime}$ potential energies computed at the data points using 50 PIP-BNN samples (red, Subfig. S11a) and 3000 PIP-BNN samples (red, Subfig. S11b), compared to the energies resulting from Varga et 15 fits (green dots). 


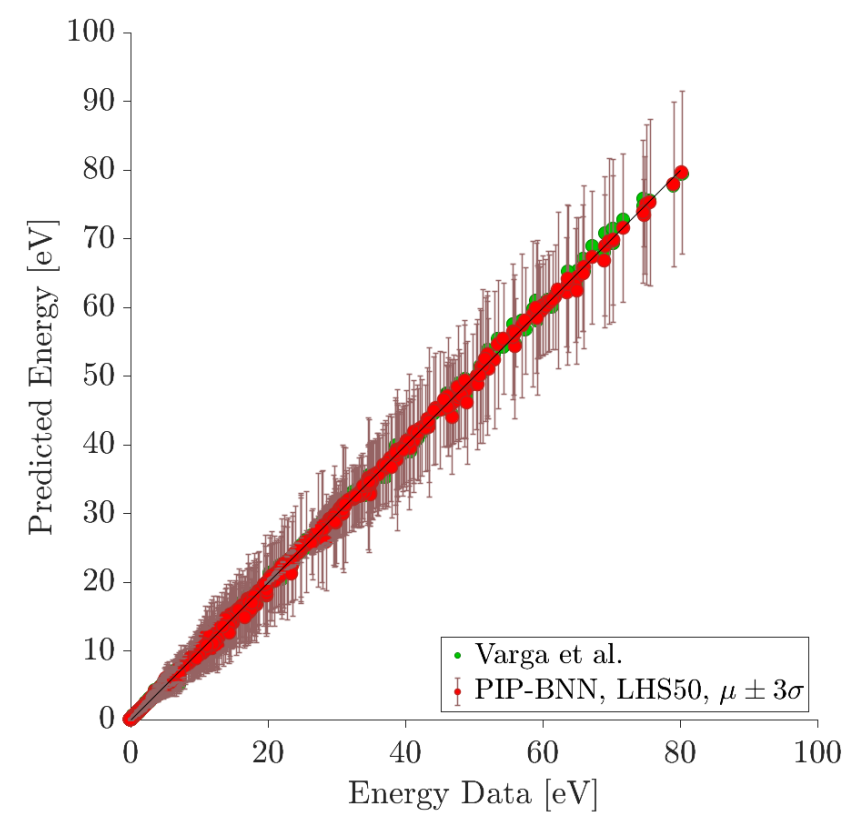

(a)

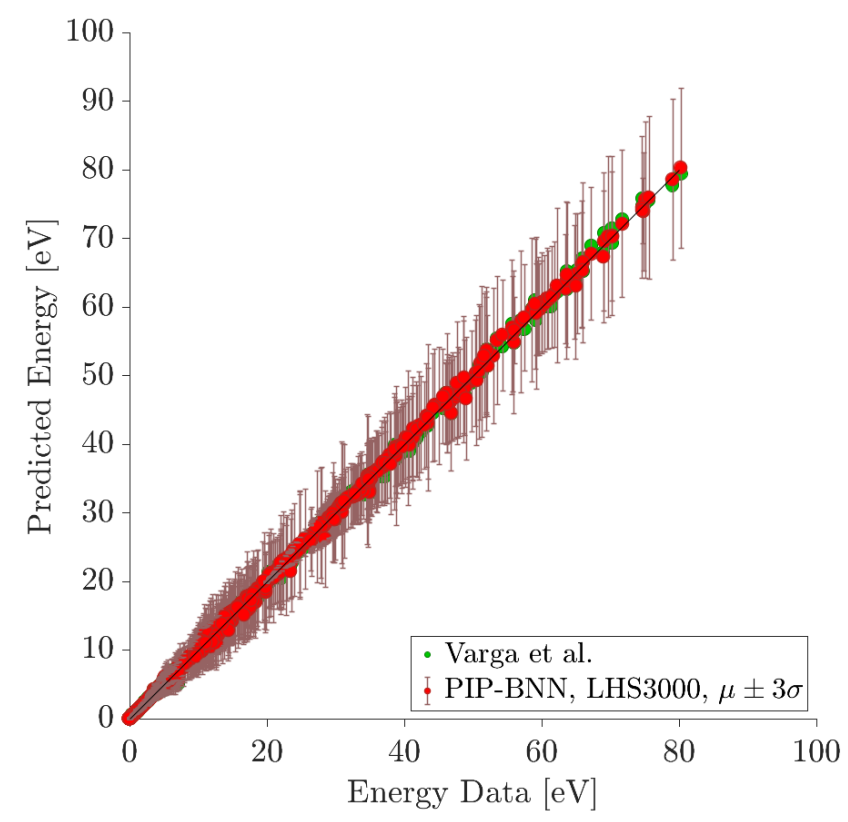

(b)

FIG. S12: Means and three-sigma confidence intervals of the $2^{5} A^{\prime}$ potential energies computed at the data points using 50 PIP-BNN samples (red, Subfig. S12a) and 3000 PIP-BNN samples (red, Subfig. S12b), compared to the energies resulting from Varga et al.'s fits (green dots). 


\section{B. PESs 2D Cuts}

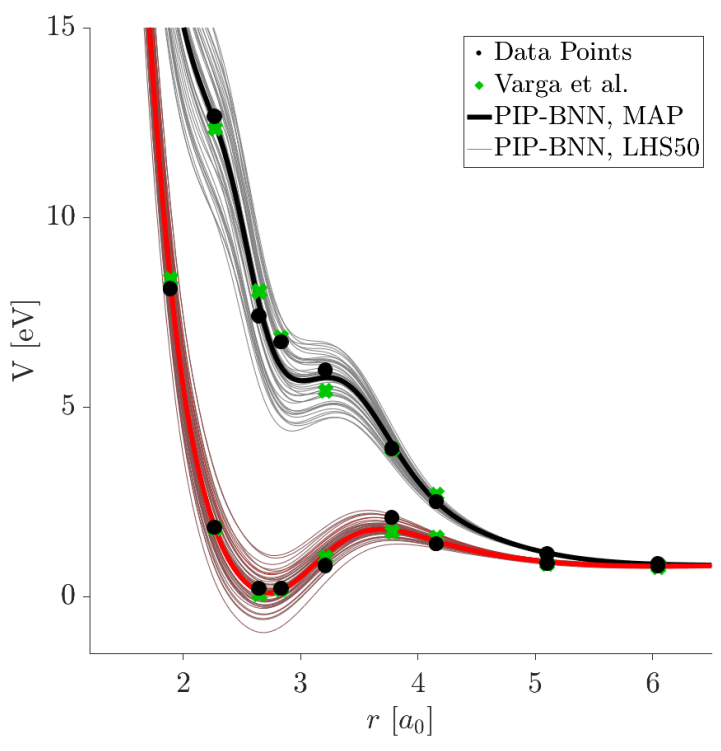

(a) $\measuredangle O_{A} O_{B} O_{C}=60^{\circ}$ and $r_{1}=2.646 a_{0}$

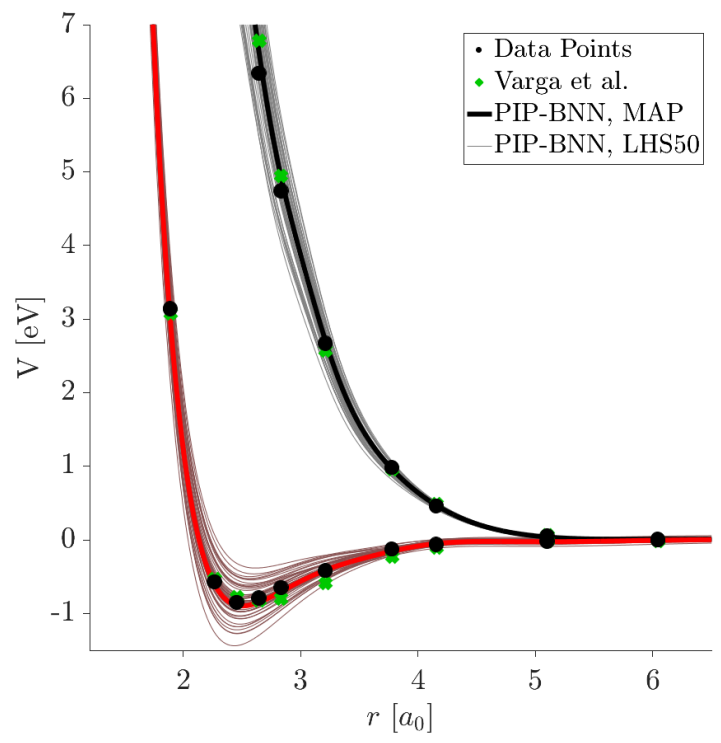

(c) $\measuredangle O_{A} O_{B} O_{C}=110^{\circ}$ and $r_{1}=2.268 a_{0}$

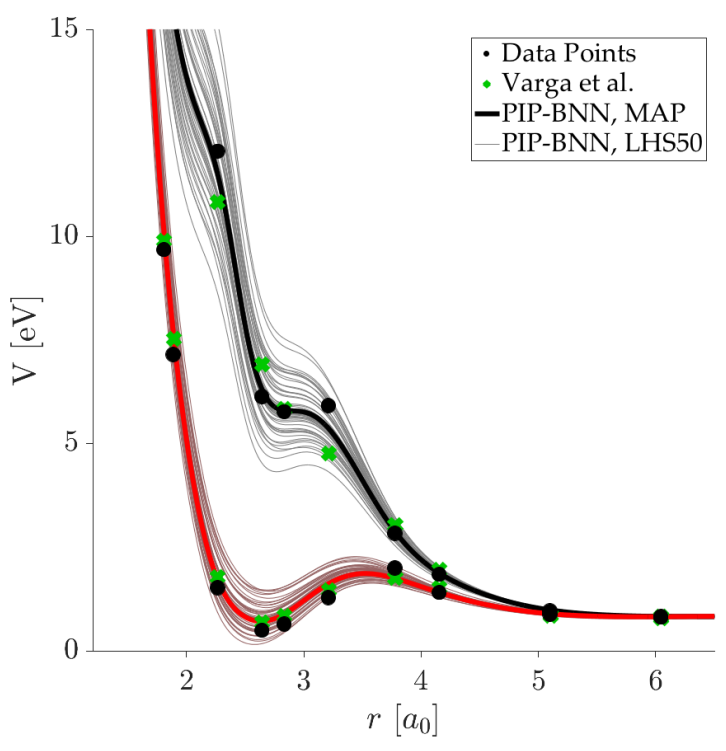

(b) $\measuredangle O_{A} O_{B} O_{C}=70^{\circ}$ and $r_{1}=2.646 a_{0}$

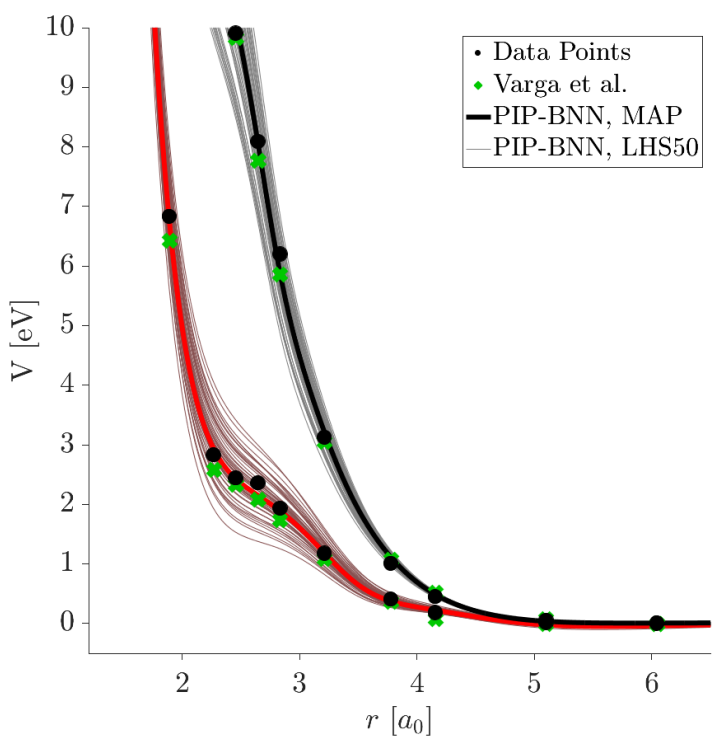

(d) $\measuredangle O_{A} O_{B} O_{C}=170^{\circ}$ and $r_{1}=2.268 a_{0}$

FIG. S13: $1^{1} A^{\prime}$ (red lower parts of the Fig.s) and $2^{5} A^{\prime}$ (black upper parts) PESs at four different sets of geometries. Black dots correspond to the ab initio data points, green crosses to Varga et al.'s fit at the data geometries, bold lines to PIP-BNN MAP, and the remaining narrow lines to the 50 PIP-BNN samples. 


\section{PESs 3D Cuts}

\section{Results for $1^{1} A^{\prime}$ PESS}
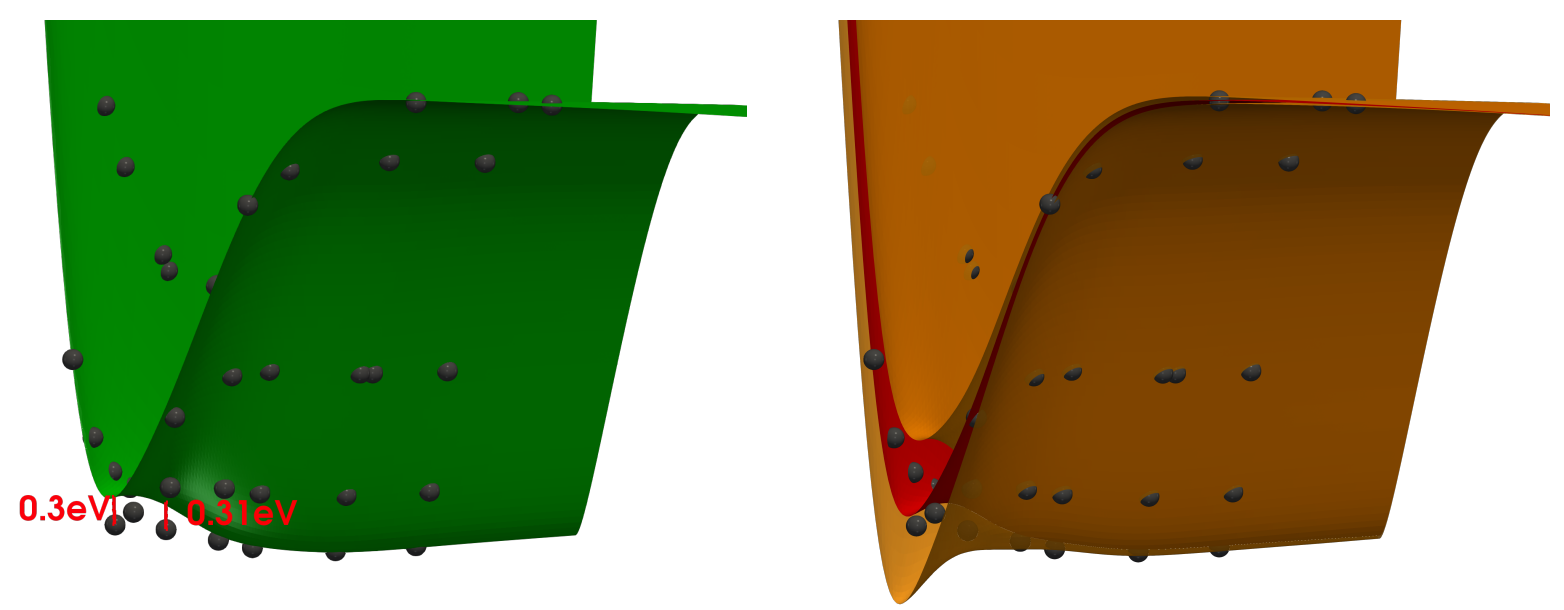

(a) $\measuredangle O_{A} O_{B} O_{C}=90^{\circ}$, Varga et al.

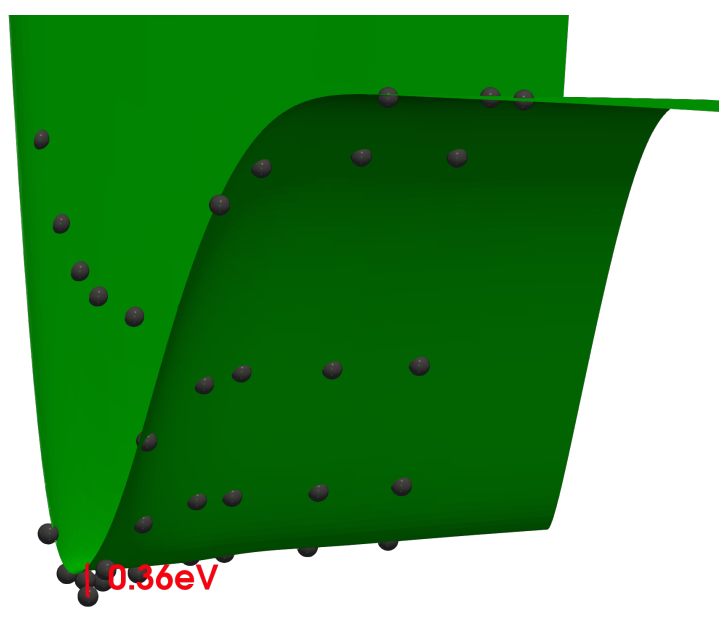

(b) $\measuredangle O_{A} O_{B} O_{C}=90^{\circ}$, PIP-BNN

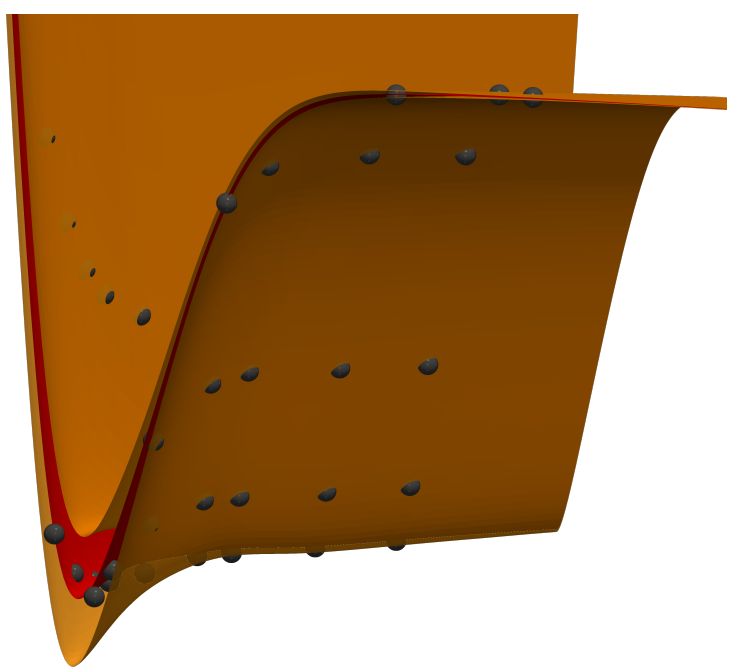

(c) $\measuredangle O_{A} O_{B} O_{C}=100^{\circ}$, Varga et al.

(d) $\measuredangle O_{A} O_{B} O_{C}=100^{\circ}$, PIP-BNN

FIG. S14: $3 \mathrm{D}$ views of the $1^{1} A^{\prime}$ PESs at $r_{1}>r_{3}$ and multiple $\measuredangle O_{A} O_{B} O_{C}$, compared to the ab initio data points (black dots). The green surface in Subfig. ?? represents Varga et al.'s fit, the red one identifies the mean of the 50 PIP-BNN samples, while the orange ones correspond to the bounds of the three-sigma confidence intervals generated by such samples. 


\section{Results for $2^{5} A^{\prime} P E S s$}
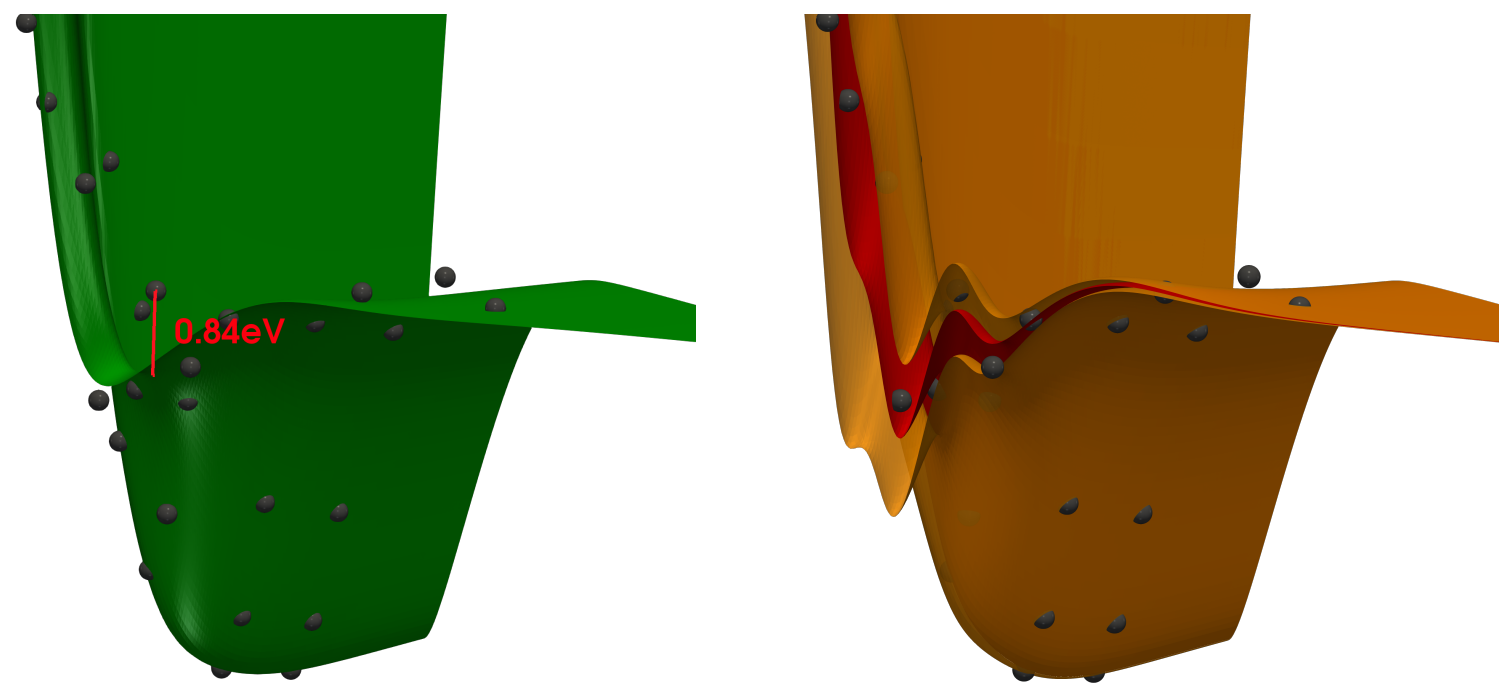

(a) $\measuredangle O_{A} O_{B} O_{C}=60^{\circ}$, Varga et al.

(b) $\measuredangle O_{A} O_{B} O_{C}=60^{\circ}$, PIP-BNN
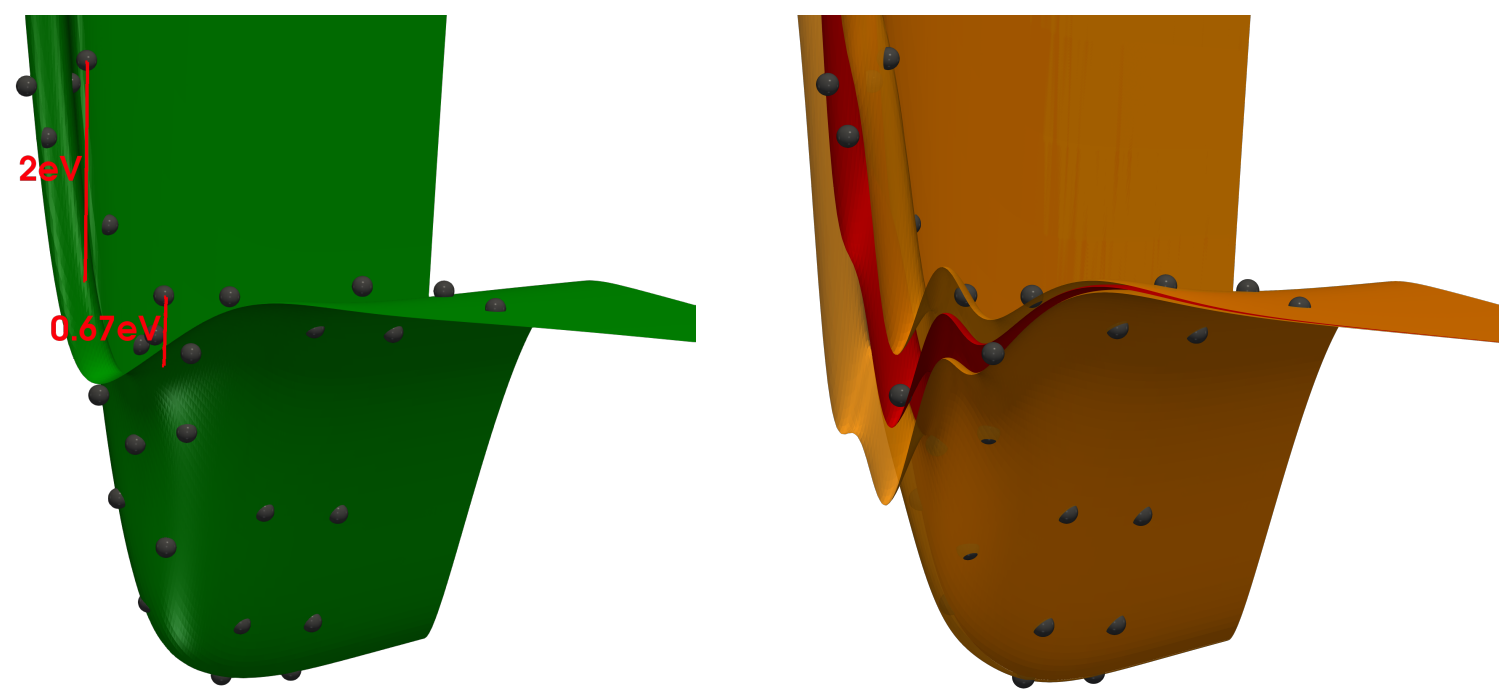

(c) $\measuredangle O_{A} O_{B} O_{C}=65^{\circ}$, Varga et al.

(d) $\measuredangle O_{A} O_{B} O_{C}=65^{\circ}$, PIP-BNN

FIG. S15: $3 \mathrm{D}$ views of the $2^{5} A^{\prime}$ PESs at $r_{1}>r_{3}$ and multiple $\measuredangle O_{A} O_{B} O_{C}$, compared to the ab initio data points (black dots). The green surfaces represent Varga et al.'s fit, the red ones identifies the mean of the 50 PIP-BNN samples, while the orange ones correspond to the bounds of the three-sigma confidence intervals generated by such samples. 


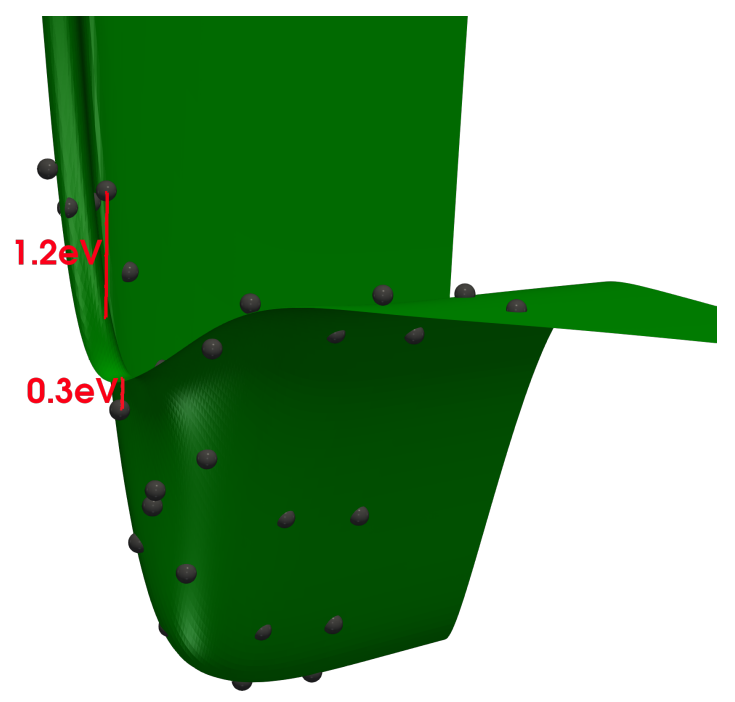

(a) $\measuredangle O_{A} O_{B} O_{C}=70^{\circ}$, Varga et al.

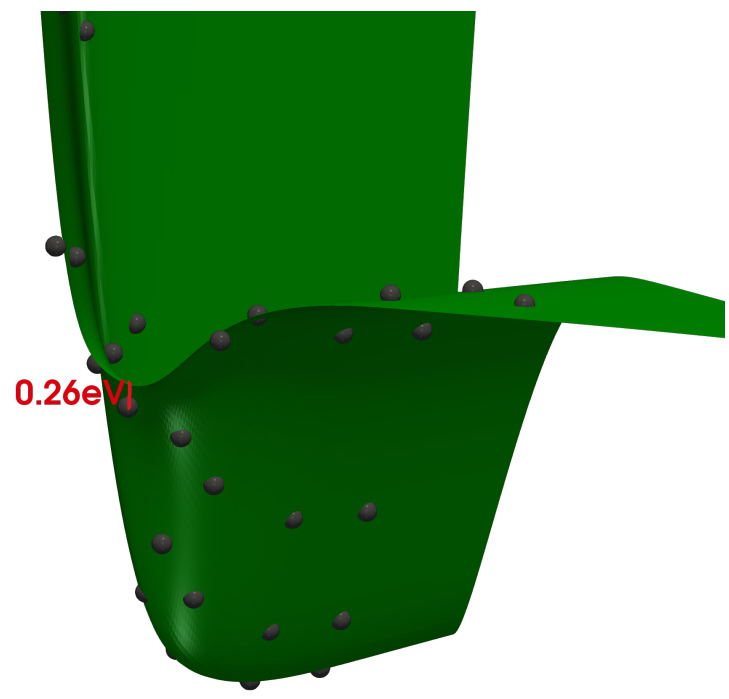

(c) $\measuredangle O_{A} O_{B} O_{C}=80^{\circ}$, Varga et al.

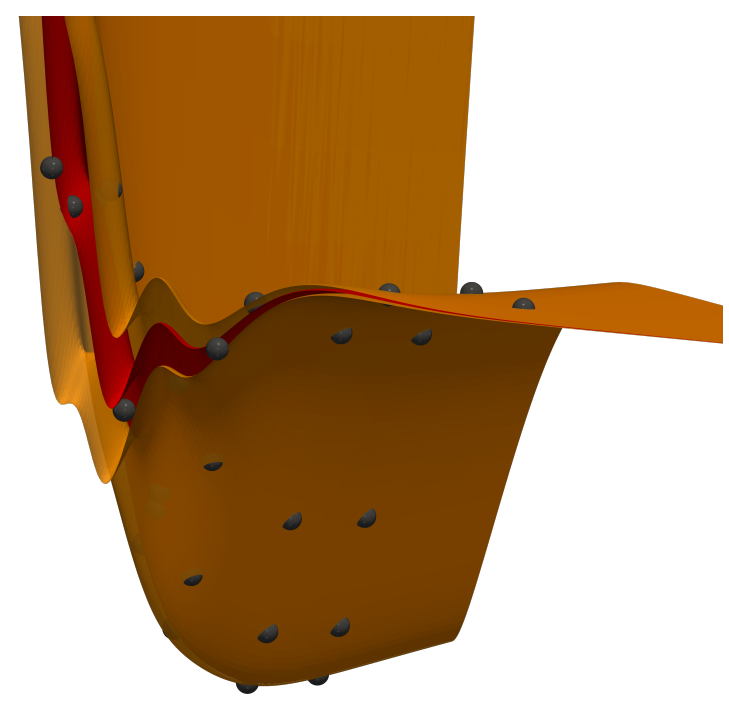

(b) $\measuredangle O_{A} O_{B} O_{C}=70^{\circ}$, PIP-BNN

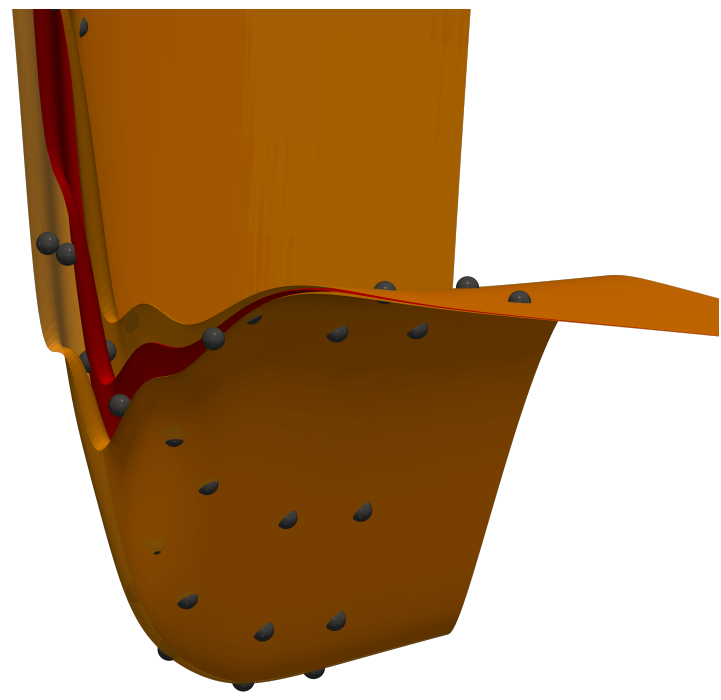

(d) $\measuredangle O_{A} O_{B} O_{C}=80^{\circ}$, PIP-BNN

FIG. S16: $3 \mathrm{D}$ views of the $2^{5} A^{\prime} \mathrm{PESs}$ at $r_{1}>r_{3}$ and multiple $\measuredangle O_{A} O_{B} O_{C}$, compared to the ab initio data points (black dots). The green surfaces represent Varga et al.'s fit, the red ones identifies the mean of the 50 PIP-BNN samples, while the orange ones correspond to the bounds of the three-sigma confidence intervals generated by such samples. 


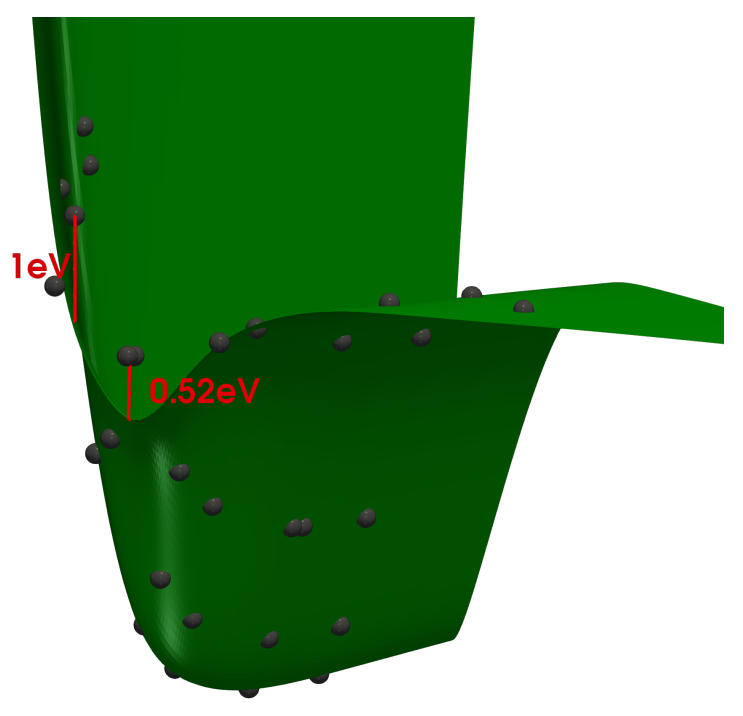

(a) $\measuredangle O_{A} O_{B} O_{C}=90^{\circ}$, Varga et al.

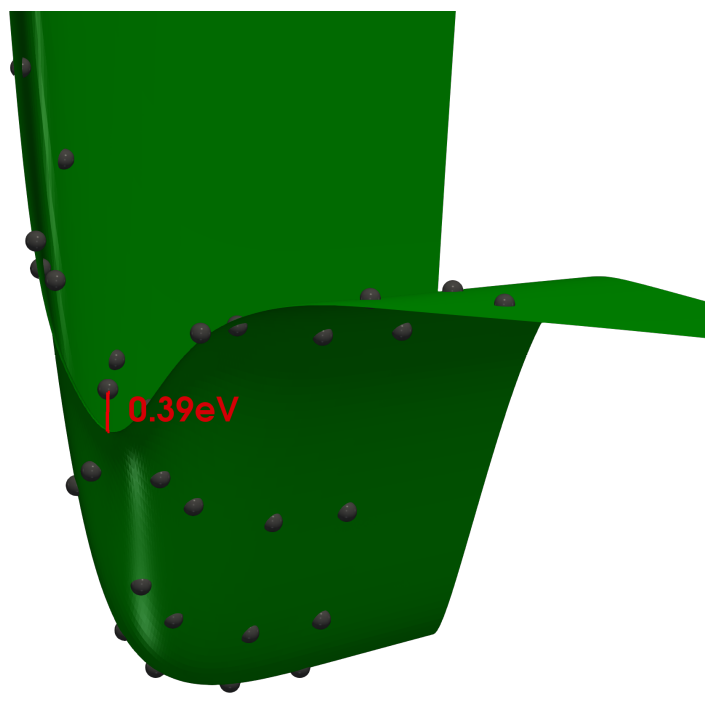

(c) $\measuredangle O_{A} O_{B} O_{C}=100^{\circ}$, Varga et al.

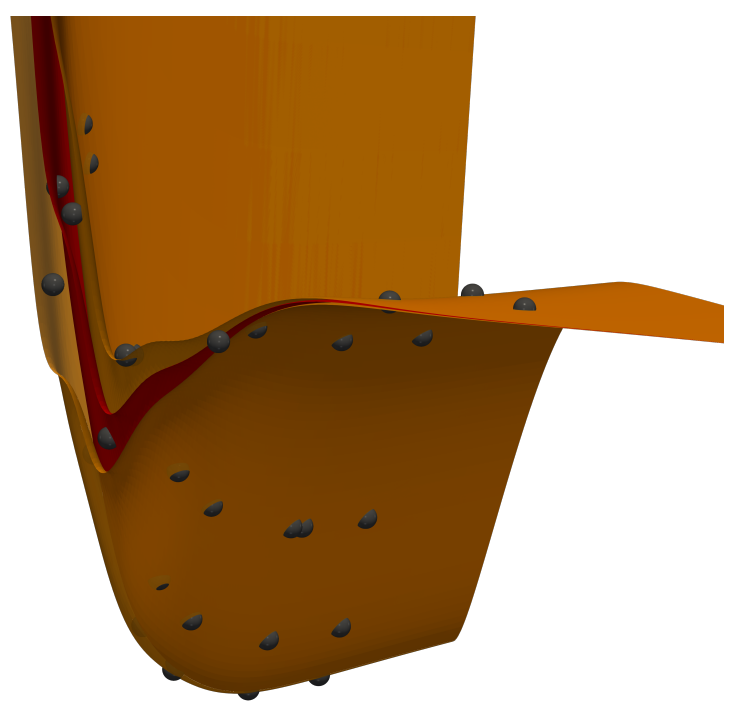

(b) $\measuredangle O_{A} O_{B} O_{C}=90^{\circ}$, PIP-BNN

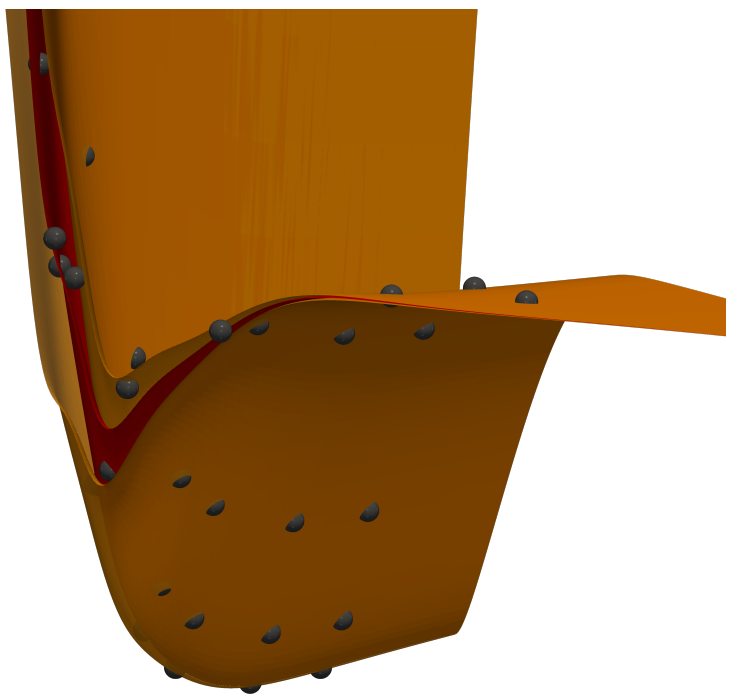

(d) $\measuredangle O_{A} O_{B} O_{C}=100^{\circ}$, PIP-BNN

FIG. S17: $3 \mathrm{D}$ views of the $2^{5} A^{\prime}$ PESs at $r_{1}>r_{3}$ and multiple $\measuredangle O_{A} O_{B} O_{C}$, compared to the ab initio data points (black dots). The green surfaces represent Varga et al.'s fit, the red ones identifies the mean of the 50 PIP-BNN samples, while the orange ones correspond to the bounds of the three-sigma confidence intervals generated by such samples. 


\section{Rate Coefficients at $\mathbf{T}=\mathbf{1 0 0 0 0} \mathrm{K}$}

1. Results for $2^{5} A^{\prime}$ PESS

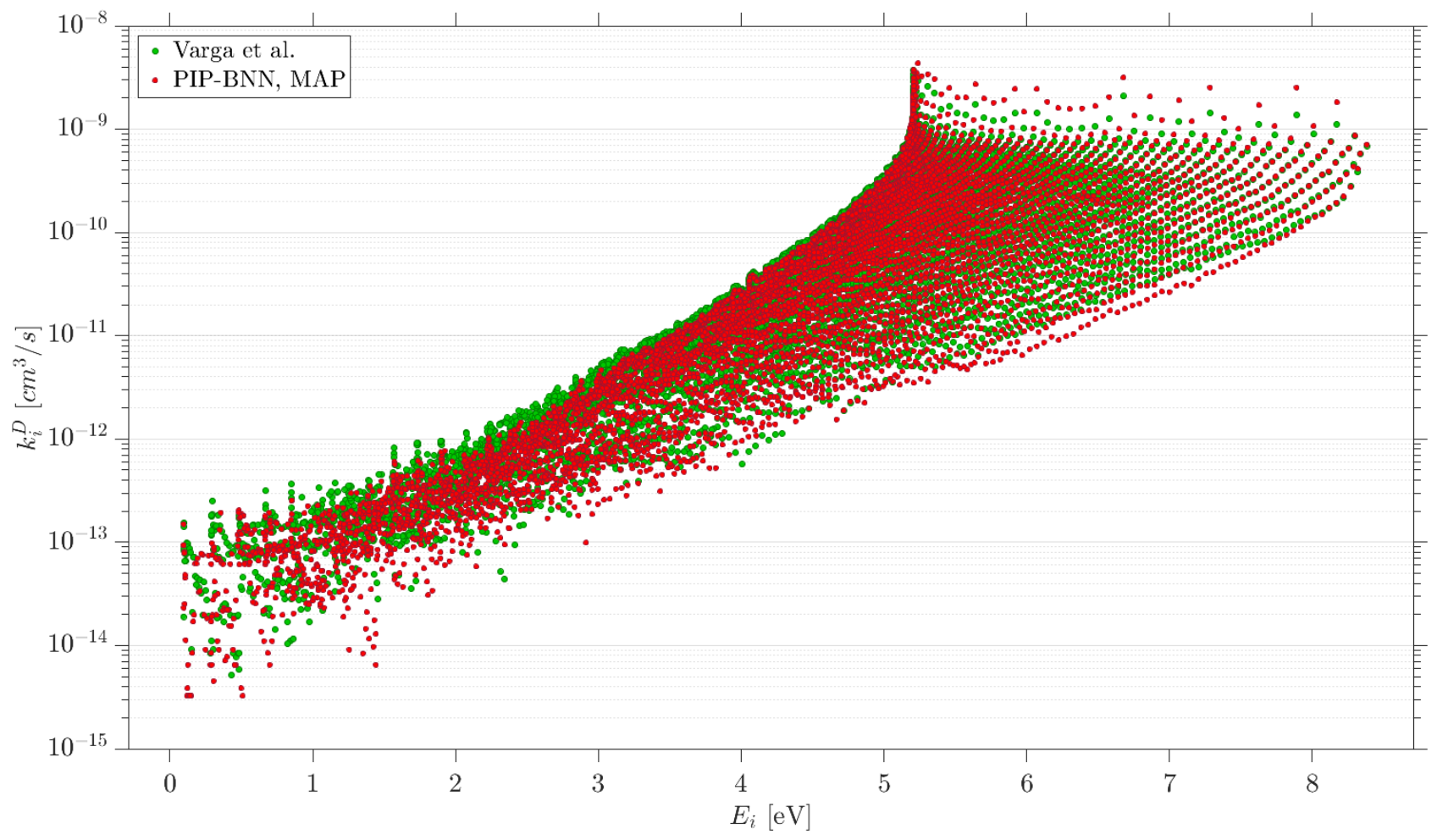

FIG. S18: Rovibrational state specific dissociation rate coefficients for the $2^{5} A^{\prime}$ interactions at

$T_{T r a n}=10000 \mathrm{~K}$. Green dots are obtained using Varga et al.'s fit, while the red ones are computed through the PIP-BNN MAP surface. 


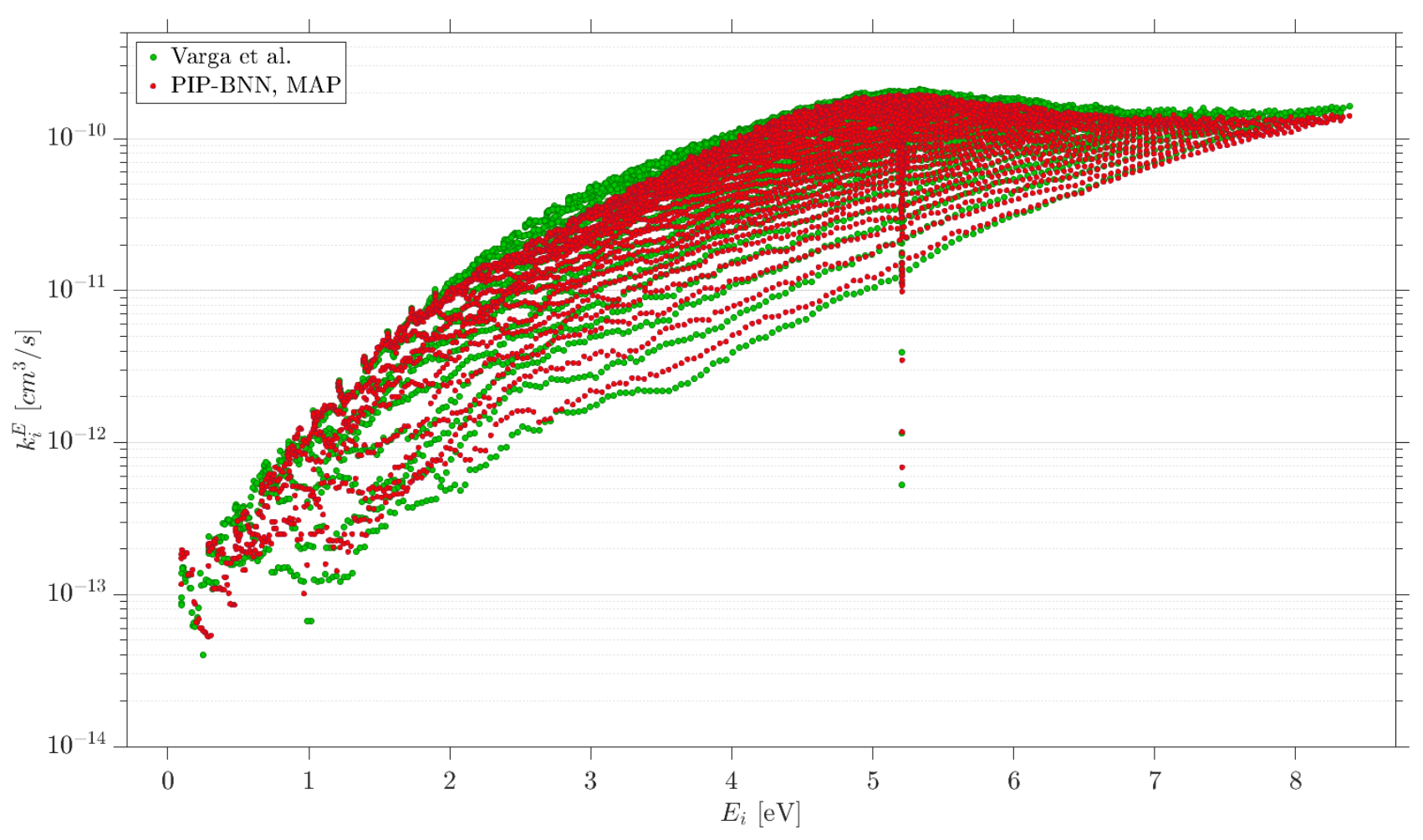

FIG. S19: Rovibrational state specific exchange rate coefficients for the $2^{5} A^{\prime}$ interactions at $T_{\text {Tran }}=10000 \mathrm{~K}$, computed as $k_{i}^{E}=\sum_{j=1}^{N_{R V}} k_{i j}^{E}$, where $i$ corresponds to the initial level, $j$ to the final one, and $N_{R V}$ to the number of $\mathrm{O}_{2}$ ro-vibrational level. Green dots are obtained using Varga et al.'s fit, while the red ones are computed through the PIP-BNN MAP surface. 


\section{E. Gas Quantities of Interest at $\mathrm{T}=10000 \mathrm{~K}$}

\section{Results for $2^{5} A^{\prime} P E S S$}

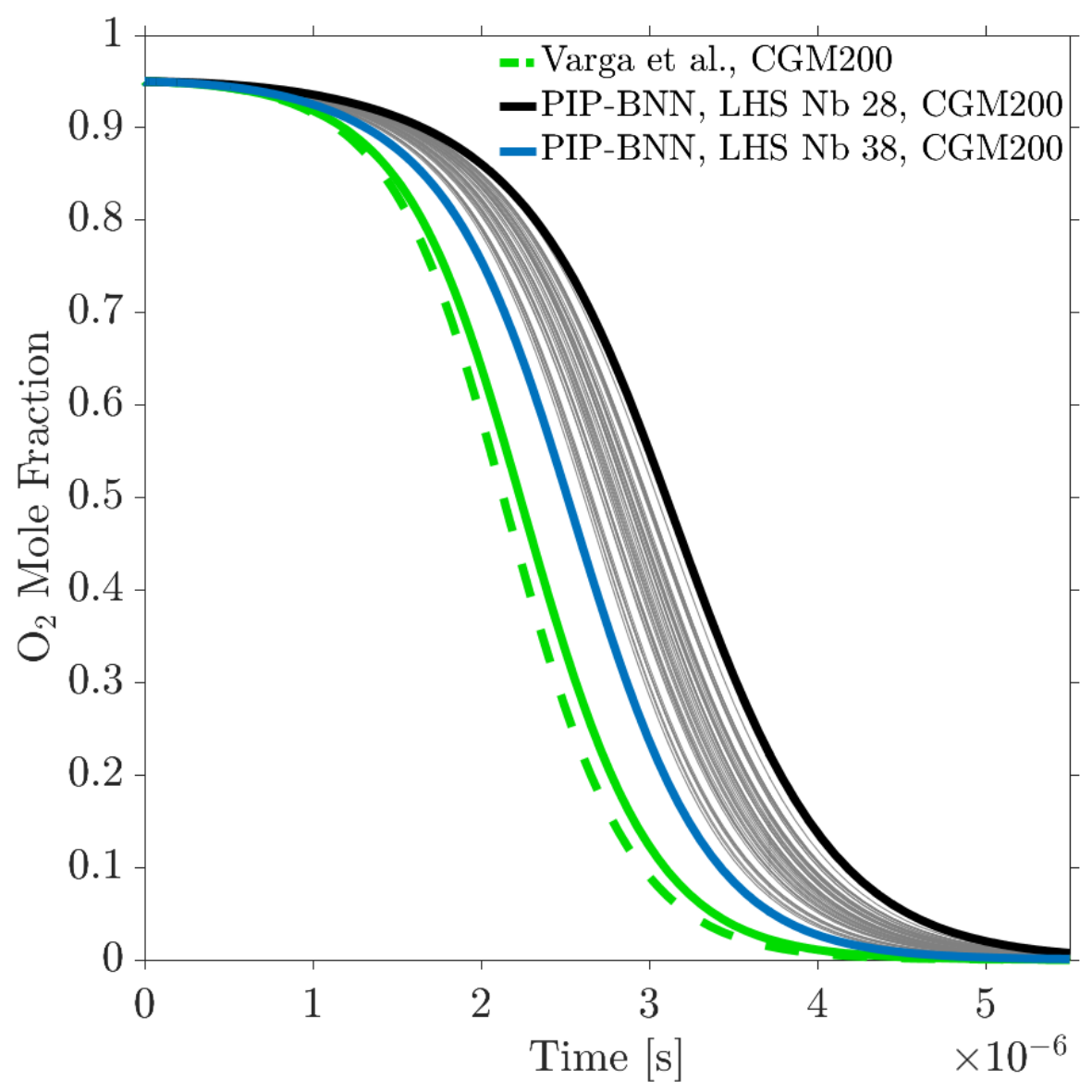

FIG. S20: Evolution of $\mathrm{O}_{2}$ mole fraction, as predicted by ME (the green solid line) and CGM200 (the overlapping green dashed line), both starting from Varga et al.'s fit, by ME from PIP-BNN MAP (the red dotted line), and by CGM200 from the 50 PIP-BNN samples (grey narrow lines). The simulations have been performed at $T_{\text {Tran }}=10000 \mathrm{~K}$ with the rates from the $2^{5} A^{\prime}$ PESs. The two curves at extremes of the distributions generated by the LHS50 are highlighted in blue and black. 


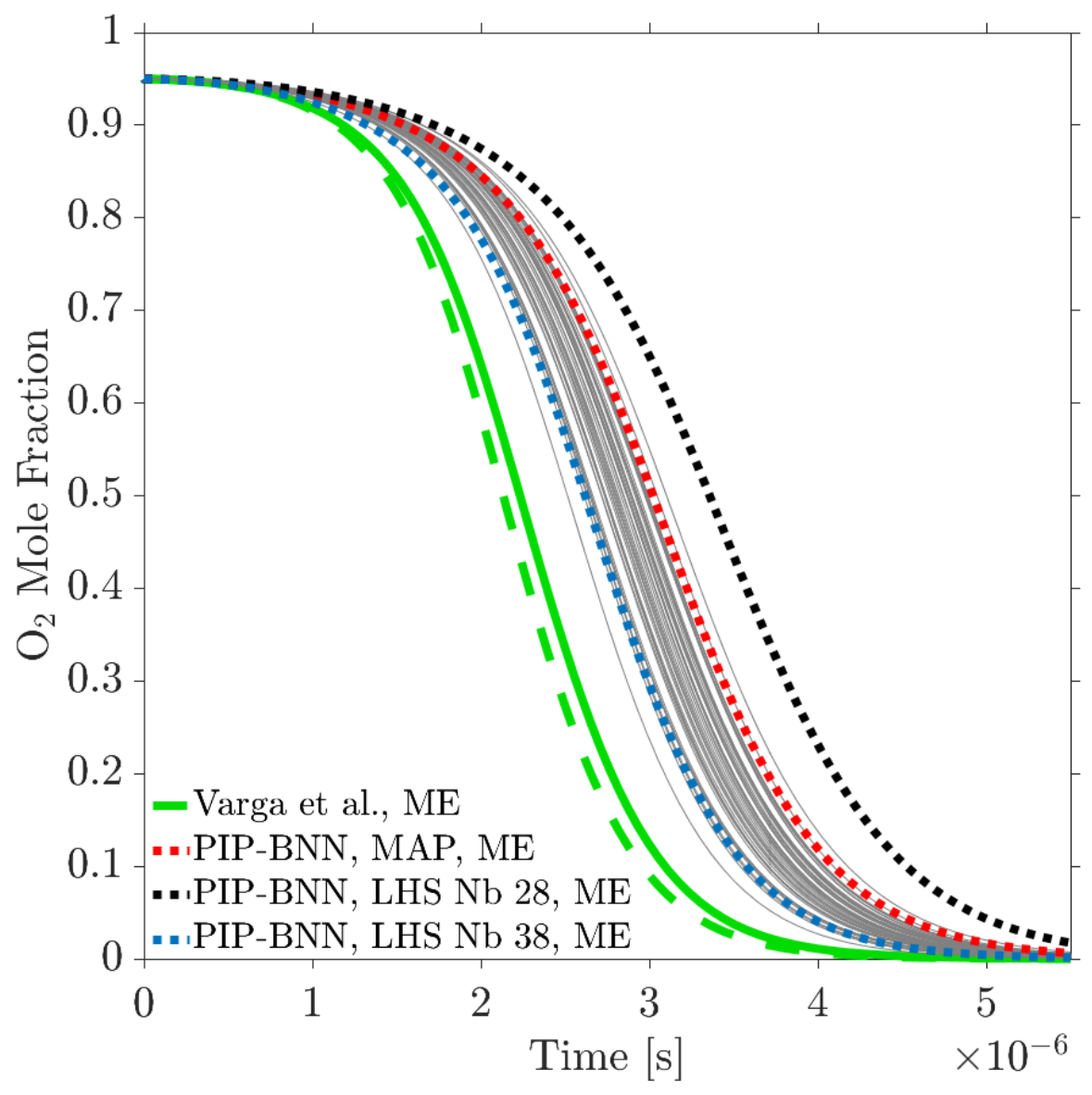

FIG. S21: Evolution of $\mathrm{O}_{2}$ dissociation global rates, as predicted by ME (green solid lines) and CGM200 (green dashed lines) using Varga et al.'s fit, by ME using the PIP-BNN MAP surface (red dotted lines), and by CGM200 using the 50 PIP-BNN samples (grey narrow lines). The Subfig.s report the histograms of the respective QSS rates. The simulations have been performed considering only $2^{5} A^{\prime}$ interactions at $T_{\text {Tran }}=10000 \mathrm{~K}$. The two LHS50 samples that generated the extremes of the distribution in the previous figure have been adopted for QCT calculations, and the resulting StS rates have been employed for ME simulations; the outcomes are reported here as blue and black dotted curves. 


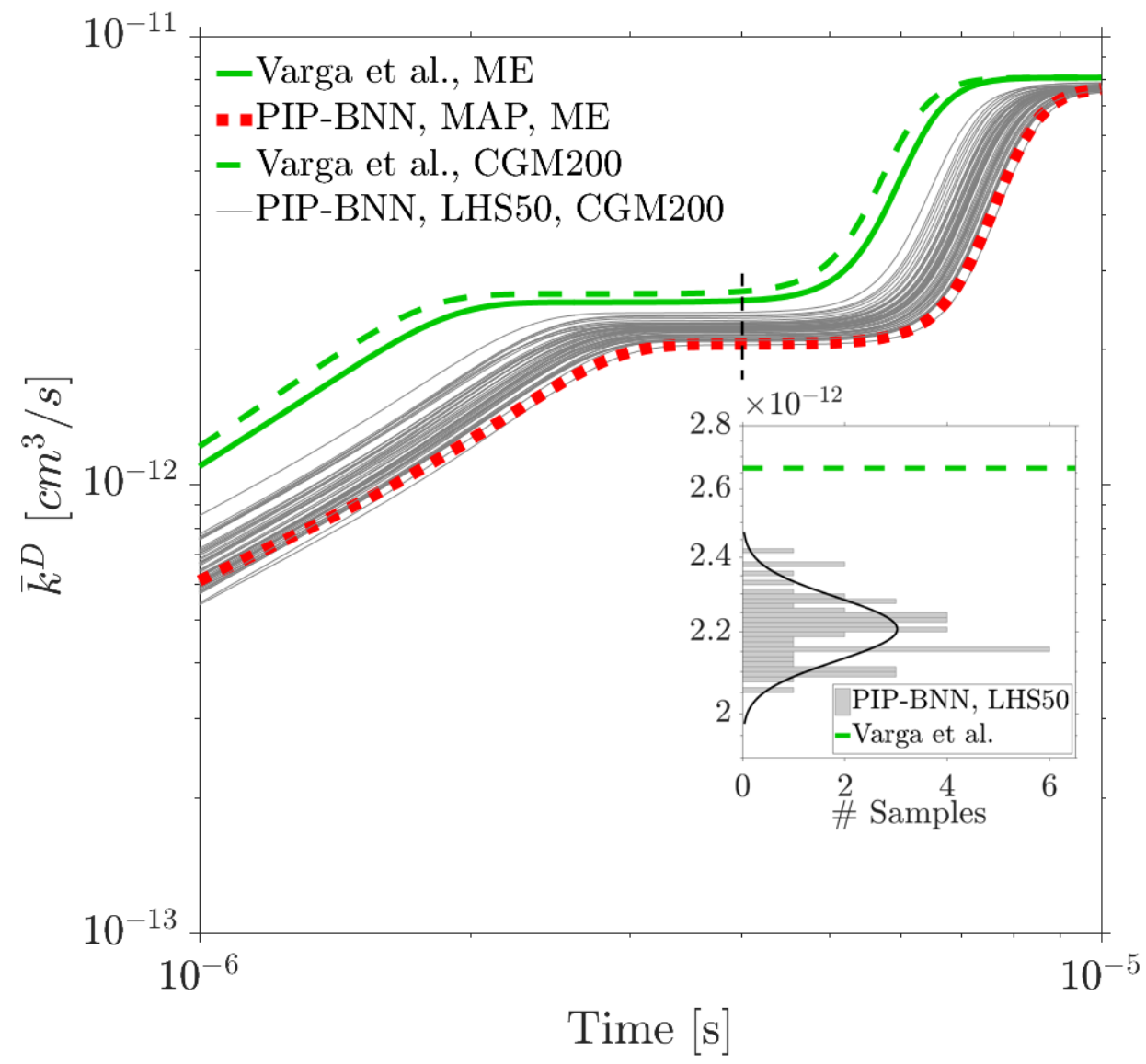

FIG. S22: Evolution of $\mathrm{O}_{2}$ dissociation global rates, as predicted by ME (green solid lines) and CGM200 (green dashed lines) using Varga et al.'s fit, by ME using the PIP-BNN MAP surface (red dotted lines), and by CGM200 using the 50 PIP-BNN samples (grey narrow lines). The Subfig.s report the histograms of the respective QSS rates. The simulations have been performed considering only $2^{5} A^{\prime}$ interactions at $T_{\text {Tran }}=10000 \mathrm{~K}$. The dissociation global rate is computed as $\overline{k^{D}}=\sum_{i} \sum_{j} k_{i}^{D} n_{i}(t) / n_{O_{2}}(t) ; i$ identifies the $\mathrm{O}_{2}$ initial state, in case of ME calculations, or the initial group in case of CGM; $k_{i}^{D}$ corresponds to the dissociation rate associated to the $\mathrm{i}$-th state/group, and $n_{i}$ represents the population of the i-th state/group. 


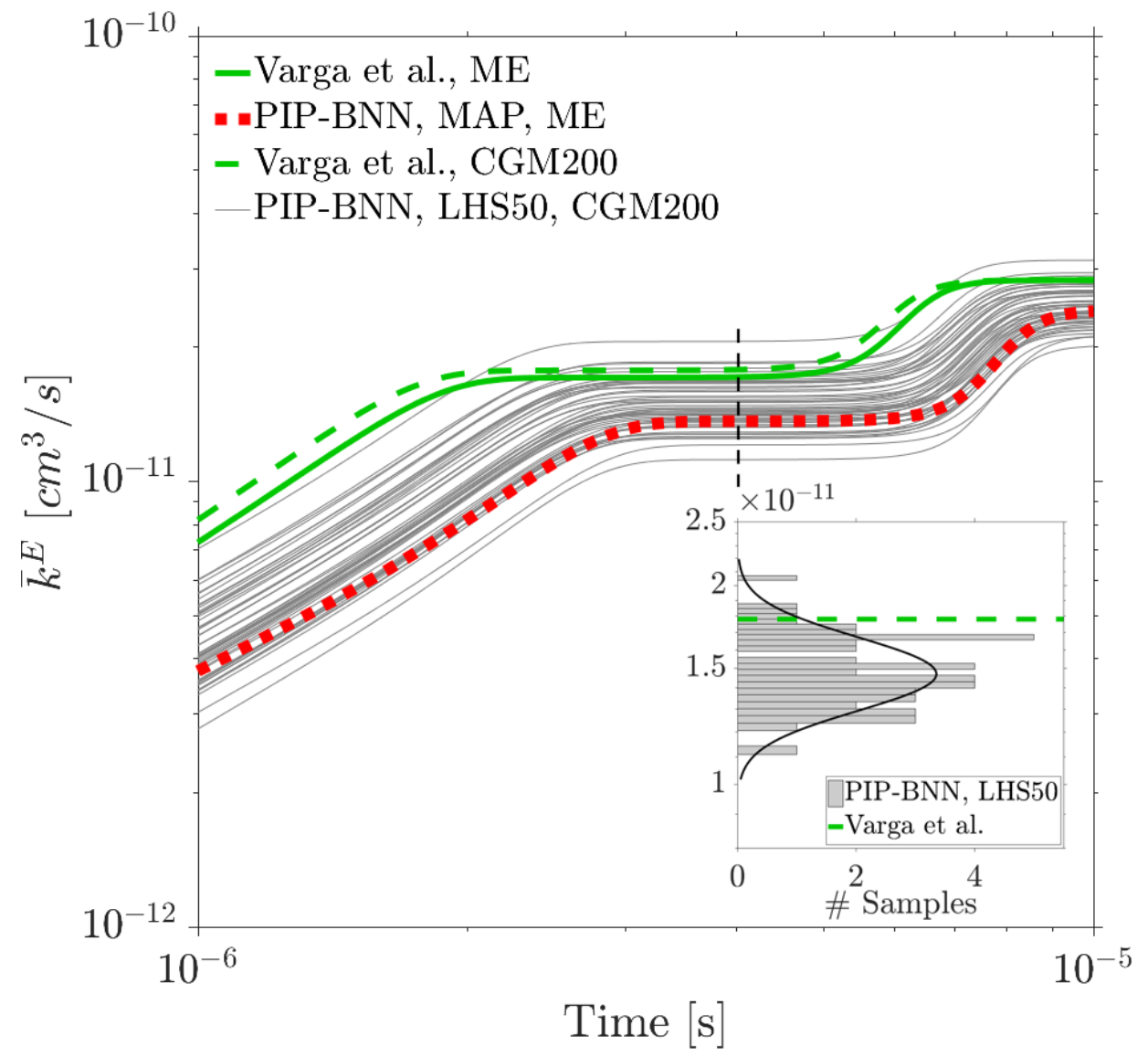

FIG. S23: Evolution of $\mathrm{O}_{2}$ exchange global rates, as predicted by ME (green solid lines) and CGM200 (green dashed lines) using Varga et al.'s fit, by ME using the PIP-BNN MAP surface (red dotted lines), and by CGM200 using the 50 PIP-BNN samples (grey narrow lines). The Subfig.s report the histograms of the respective QSS rates. The simulations have been performed considering only $2^{5} A^{\prime}$ interactions at $T_{\text {Tran }}=10000 \mathrm{~K}$. The exchange global rate is computed as $\overline{k^{E}}=\sum_{i} \sum_{j} k_{i j}^{E} n_{i}(t) / n_{O_{2}}(t) ; i$ identifies the $\mathrm{O}_{2}$ initial state, in case of ME calculations, or the initial group in case of CGM, and $j$ the final one; $k_{i j}^{E}$ corresponds to the exchange rate from the $\mathrm{i}$-th state/group to the $\mathrm{j}$-th, and $n_{i}$ represents the population of the i-th state/group. 


\section{F. Gas Quantities of Interest at $\mathbf{T}=\mathbf{2 5 0 0} \mathrm{K}$}

\section{Results for $1^{1} A^{\prime}$ PESs}

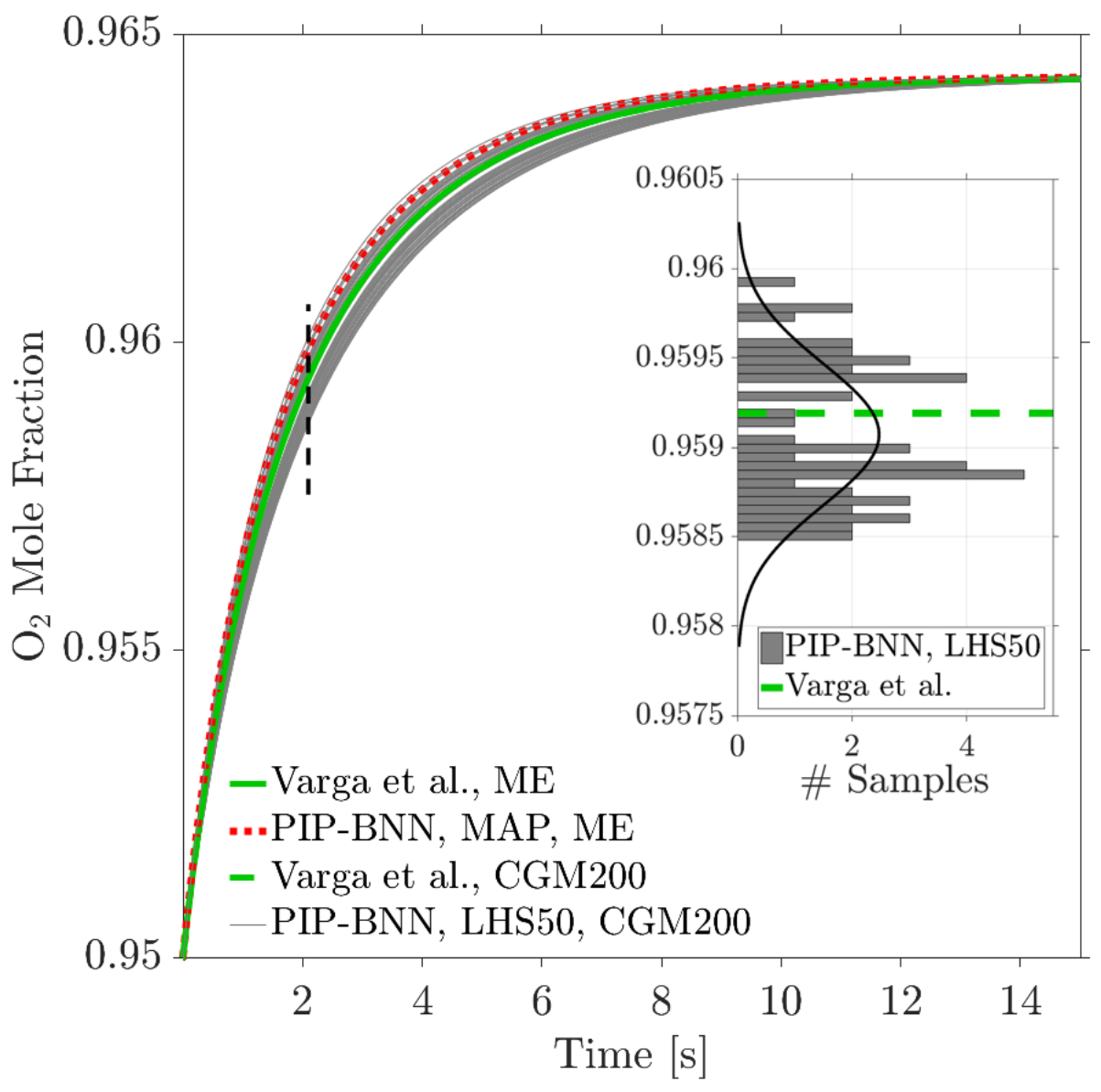

FIG. S24: Evolution of $\mathrm{O}_{2}$ mole fraction, as predicted by ME (green solid line) and CGM200 (green dashed line) using Varga et al.'s fit, by ME using the PIP-BNN MAP surface (red dotted line), and by CGM200 using the 50 PIP-BNN samples (grey narrow lines). The Subfig. on the right reports the histogram of such samples at $t=2 s$. The PIP-BNN adopted is the one constructed for $1^{1} A^{\prime}$, and the simulations are performed at $T_{\text {Tran }}=2500 \mathrm{~K}$. 


\section{Results for $2^{5} A^{\prime} P E S S$}

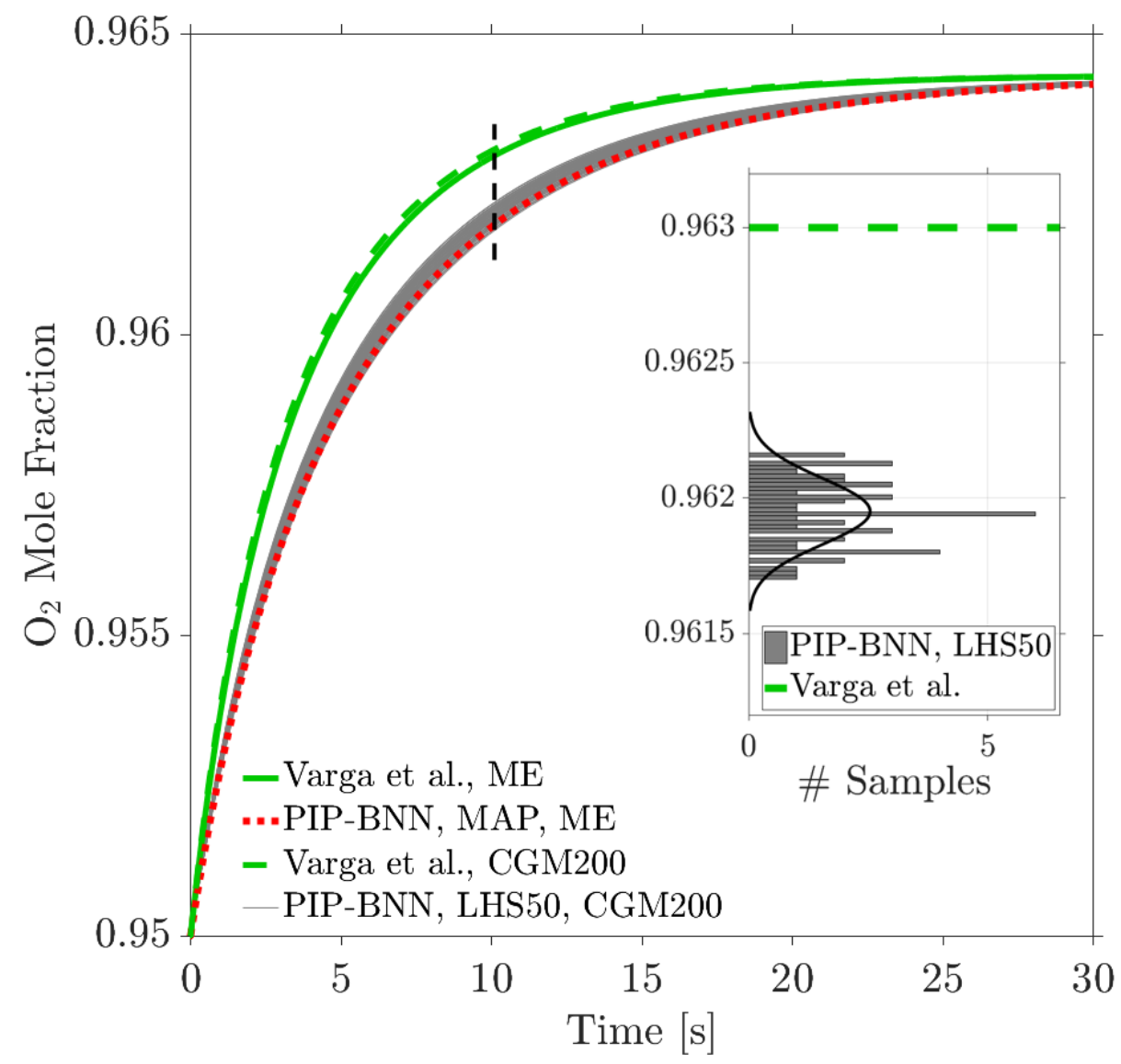

FIG. S25: Evolution of $\mathrm{O}_{2}$ mole fraction, as predicted by ME (green solid line) and CGM200 (green dashed line) using Varga et al.'s fit, by ME using the PIP-BNN MAP surface (red dotted line), and by CGM200 using the 50 PIP-BNN samples (grey narrow lines). The Subfig. on the right reports the histogram of such samples at $t=10 \mathrm{~s}$. The PIP-BNN adopted is the one constructed for $2^{5} A^{\prime}$, and the simulations are performed at $T_{\text {Tran }}=2500 \mathrm{~K}$. 


\section{G. Gas Quantities as Functions of Temperature}

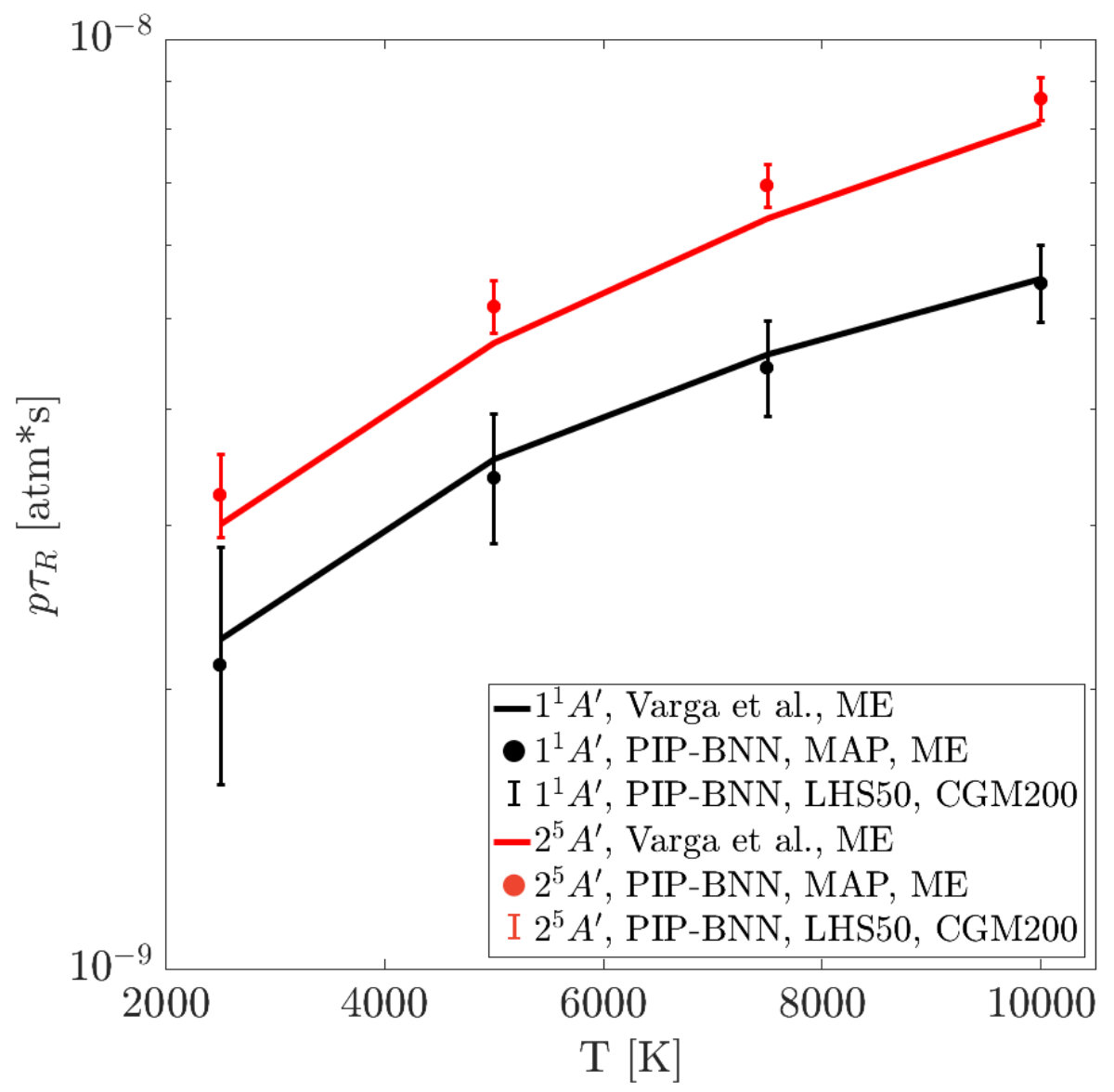

FIG. S26: Rotational relaxation times for $1^{1} A^{\prime}$ (in black) and $2^{5} A^{\prime}$ (in red) PESs. The solid lines report the values obtained using Varga et al.'s fits, the dots the ones computed through the PIP-BNN MAP surfaces, while the vertical lines identify the three-sigma intervals corresponding to the 50 PIP-BNN samples. 


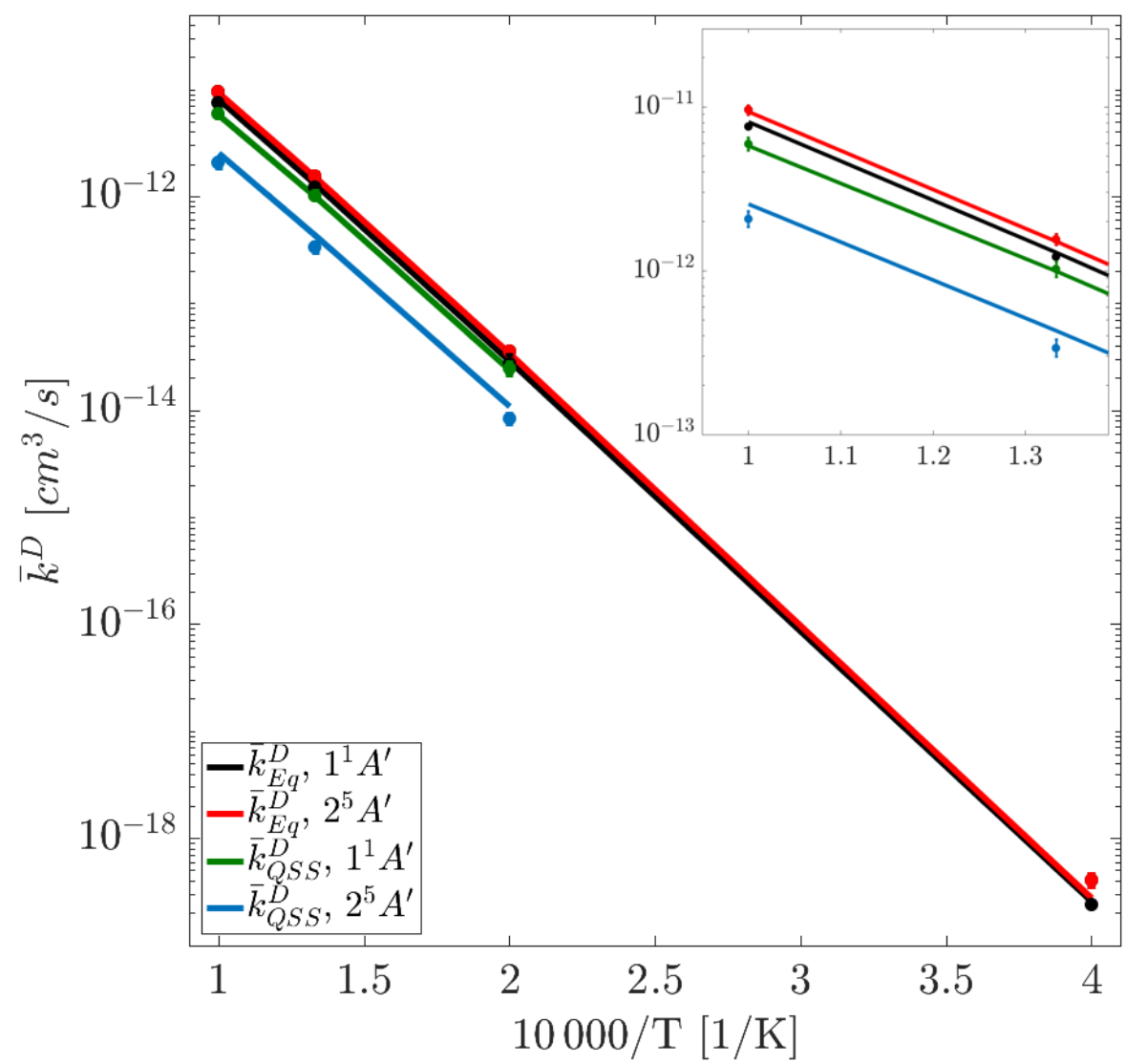

FIG. S27: Equilibrium and QSS dissociation rates for the $1^{1} A^{\prime}$ (in black and green) and $2^{5} A^{\prime}$ (in red and blue) PESs. The solid lines report the values obtained using Varga et al.'s fits, the dots the ones computed through the PIP-BNN MAP surfaces, while the vertical lines identify the three-sigma intervals corresponding to the 50 PIP-BNN samples. At $T_{T r a n}=2500 \mathrm{~K}$ no QSS is present for any of the two PESs. In the top-right square, the main Fig. is zoomed at $T_{\text {Tran }}=10000 \mathrm{~K}$ and $T_{\text {Tran }}=7500 \mathrm{~K}$. 


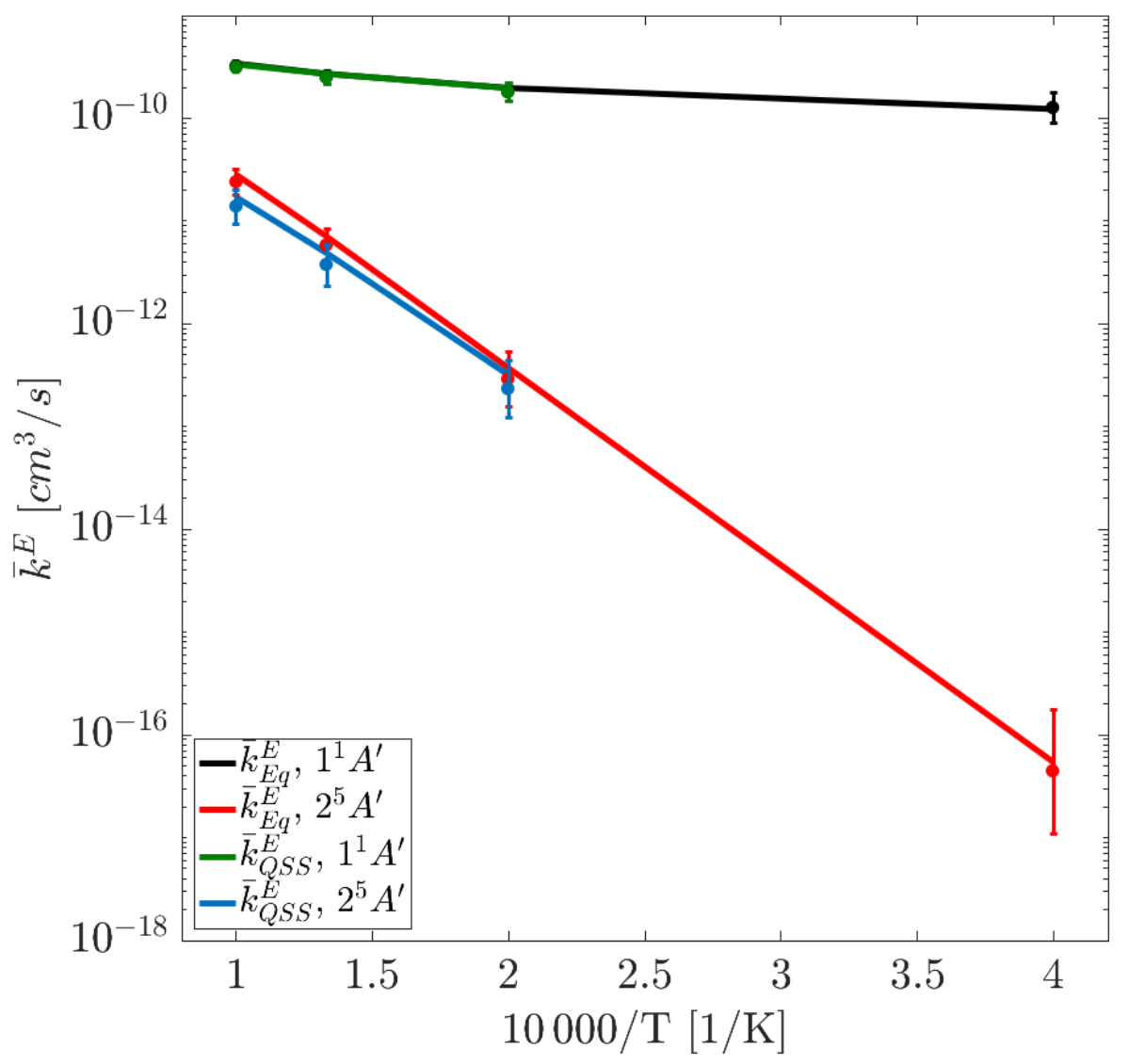

FIG. S28: Equilibrium and QSS exchange rates for the $1^{1} A^{\prime}$ (in black and green) and $2^{5} A^{\prime}$ (in red and blue) PESs. The solid lines report the values obtained using Varga et al.'s fits, the dots the ones computed through the PIP-BNN MAP surfaces, while the vertical lines identify the three-sigma intervals corresponding to the 50 PIP-BNN samples. At $T_{\text {Tran }}=2500 \mathrm{~K}$ no QSS is present for any of the two PESs. 


\section{H. Gas QoI Sensitivities to the $2^{5} A^{\prime}$ PES Uncertainties}

1. Vibrational Relaxation Times
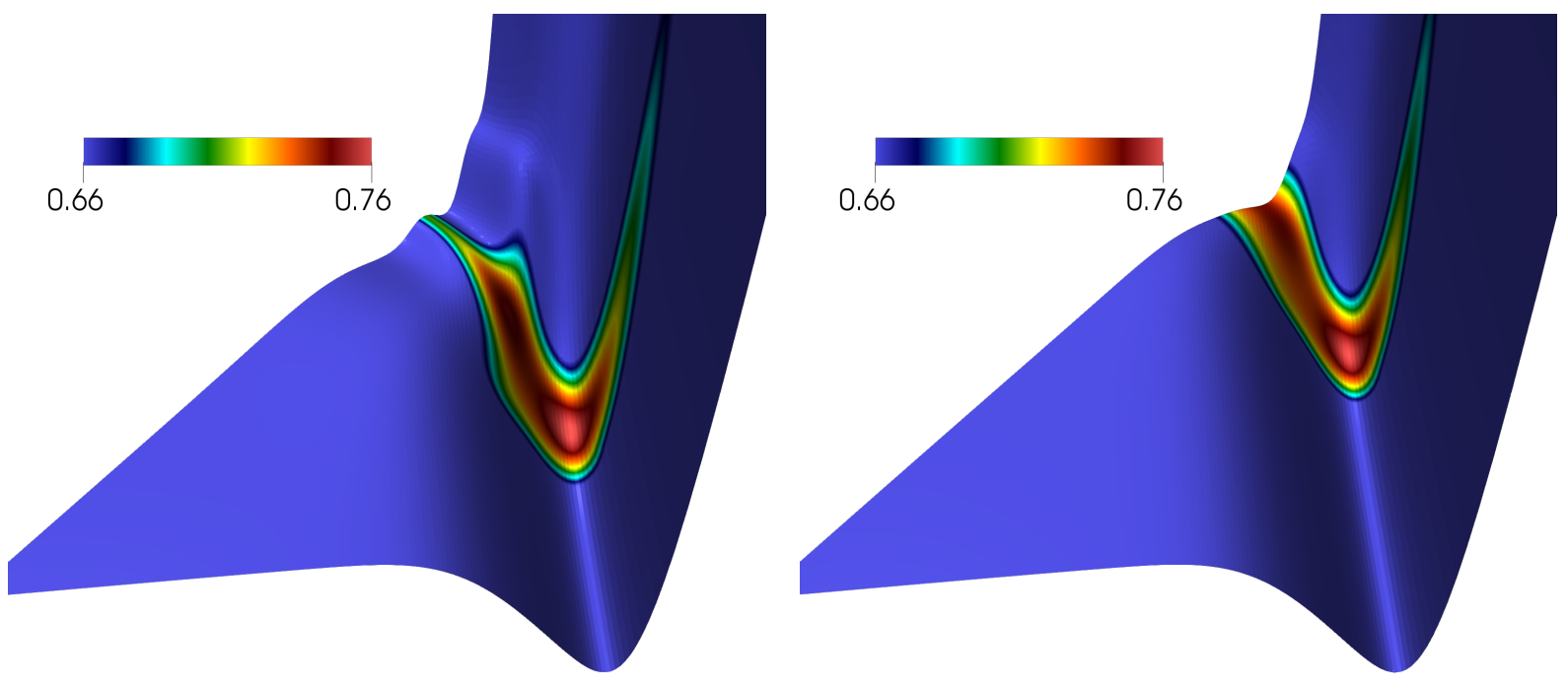

(a) $\measuredangle O_{A} O_{B} O_{C}=50^{\circ}$

(b) $\measuredangle O_{A} O_{B} O_{C}=110^{\circ}$

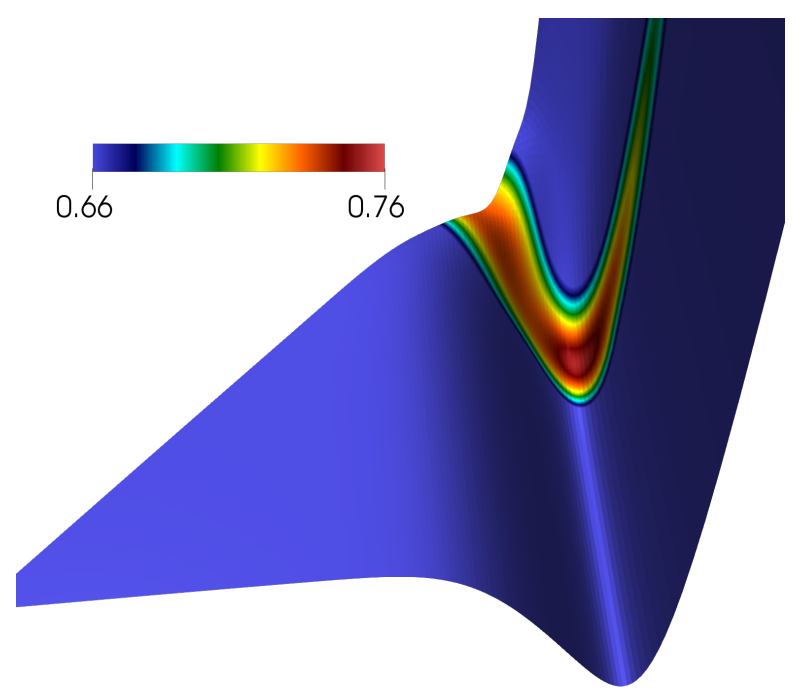

(c) $\measuredangle O_{A} O_{B} O_{C}=175^{\circ}$

FIG. S29: $3 \mathrm{D}$ views of the $2^{5} A^{\prime}$ PES resulting from the average of the 50 PIP-BNN samples for $r_{1}>r_{3}$. The surfaces have been colored based on the absolute values of Pearson correlation coefficients between the PES and the vibrational relaxation time at $T_{\text {Tran }}=10000 \mathrm{~K}$. The shades of blue in the PES depth are results of rendering effects and do not correspond to different values of Pearson coefficients. 
2. Vibrational Relaxation Times Excluding Exchange Processes
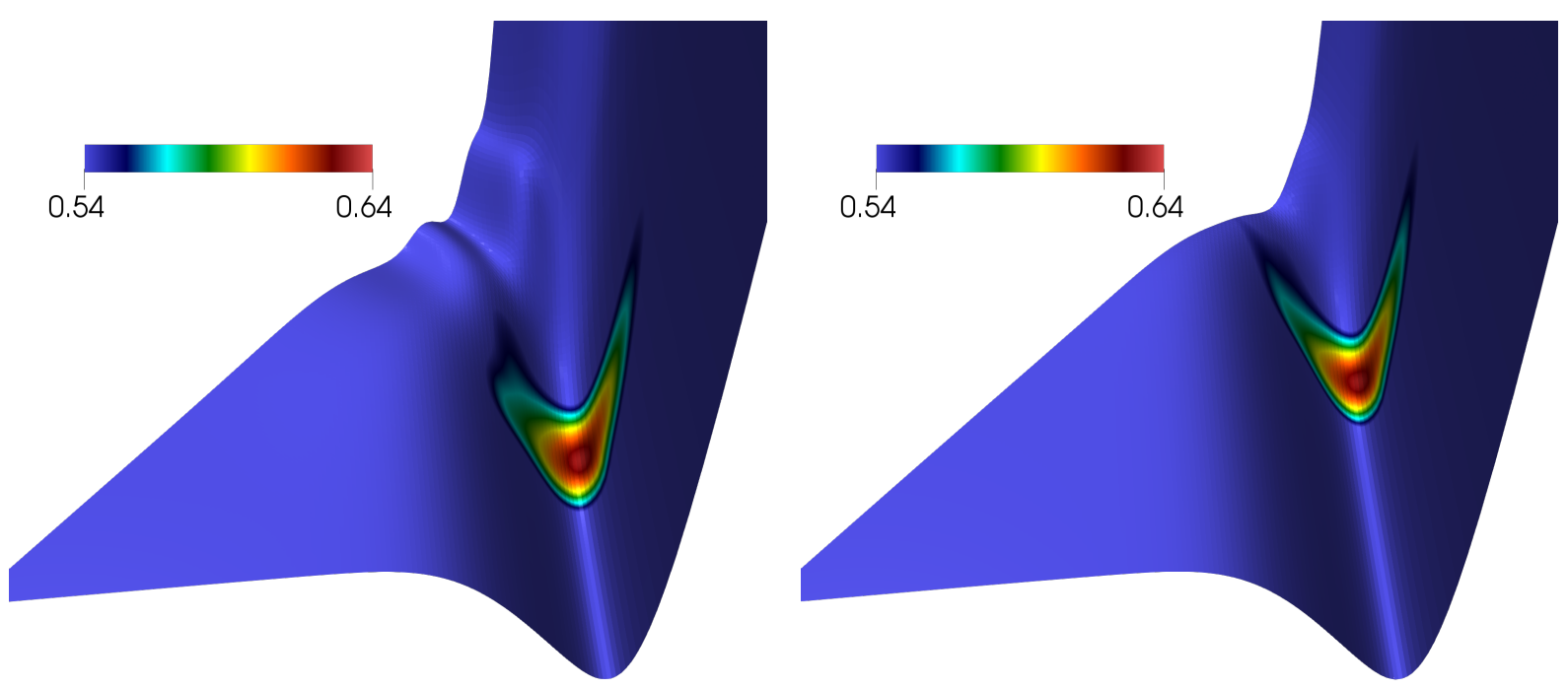

(a) $\measuredangle O_{A} O_{B} O_{C}=50^{\circ}$

(b) $\measuredangle O_{A} O_{B} O_{C}=110^{\circ}$

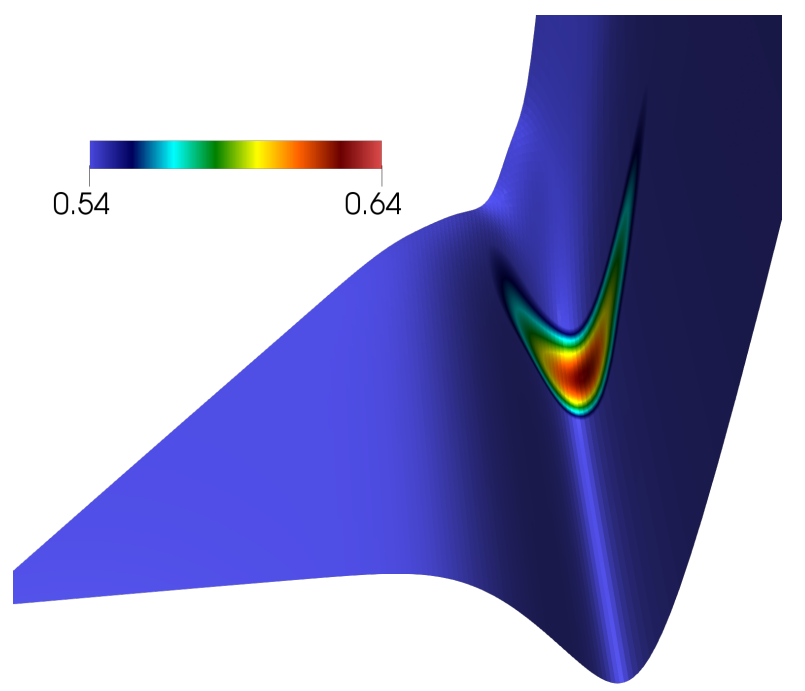

(c) $\measuredangle O_{A} O_{B} O_{C}=175^{\circ}$

FIG. S30: $3 \mathrm{D}$ views of the $2^{5} A^{\prime}$ PES resulting from the average of the 50 PIP-BNN samples for $r_{1}>r_{3}$. The surfaces have been colored based on the absolute values of Pearson correlation coefficients between the PES and the vibrational relaxation time at $T_{T r a n}=10000 \mathrm{~K}$, obtained by artificially excluding the exchange processes. The shades of blue in the PES depth are results of rendering effects and do not correspond to different values of Pearson coefficients. 


\section{Rotational Relaxation Times}
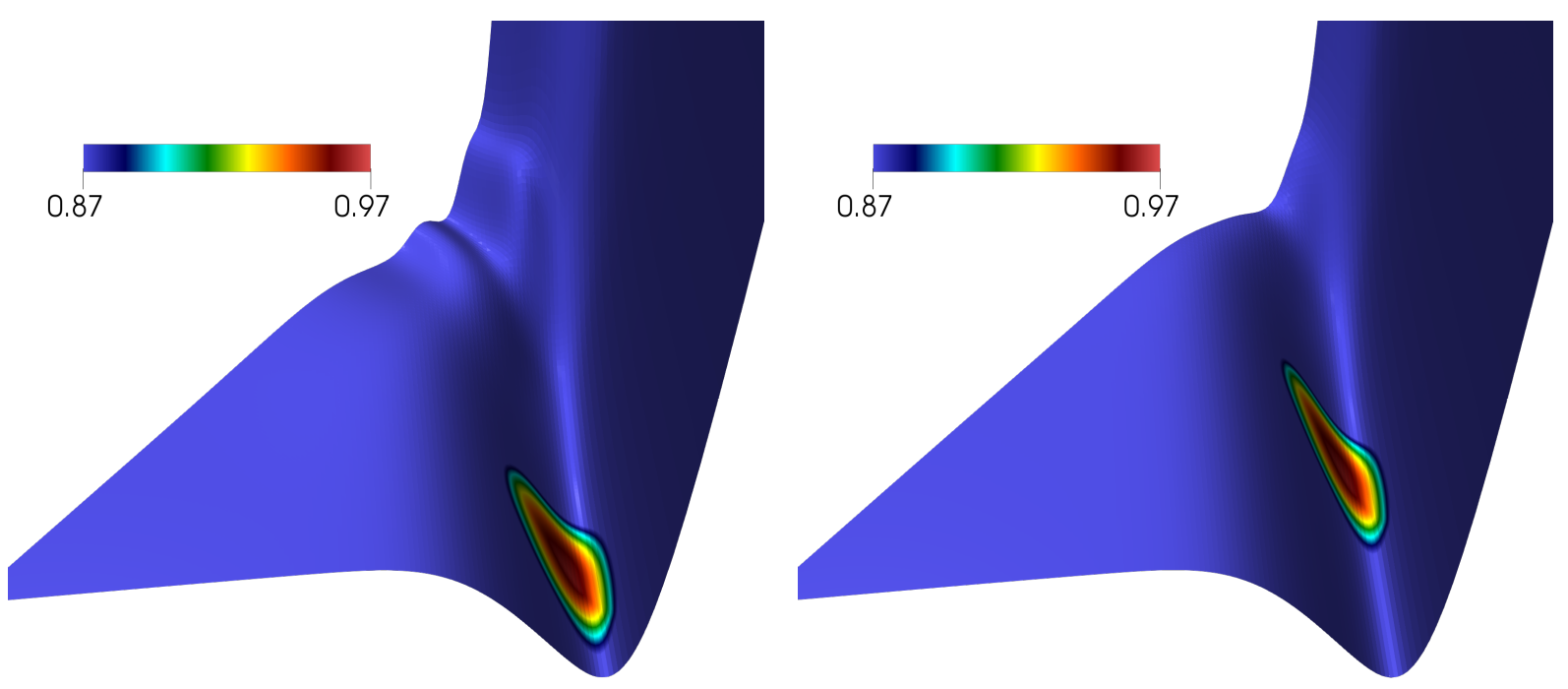

(a) $\measuredangle O_{A} O_{B} O_{C}=50^{\circ}$

(b) $\measuredangle O_{A} O_{B} O_{C}=110^{\circ}$

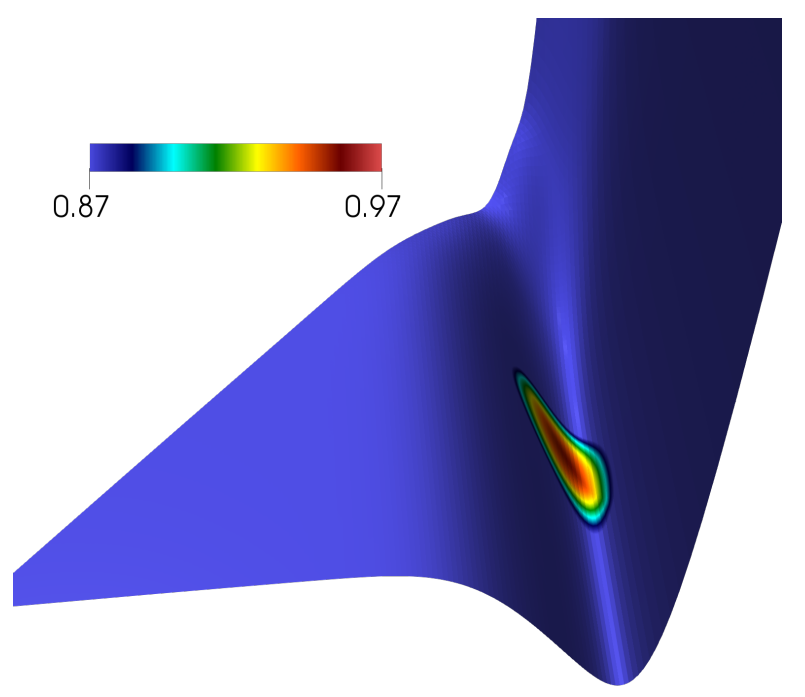

(c) $\measuredangle O_{A} O_{B} O_{C}=175^{\circ}$

FIG. S31: $3 \mathrm{D}$ views of the $2^{5} A^{\prime}$ PES resulting from the average of the 50 PIP-BNN samples for $r_{1}>r_{3}$. The surfaces have been colored based on the absolute values of Pearson correlation coefficients between the PES and the rotational relaxation time at $T_{\text {Tran }}=2500 \mathrm{~K}$. The shades of blue in the PES depth are results of rendering effects and do not correspond to different values of Pearson coefficients. 


\section{Global Dissociation Rates at Equilibrium}
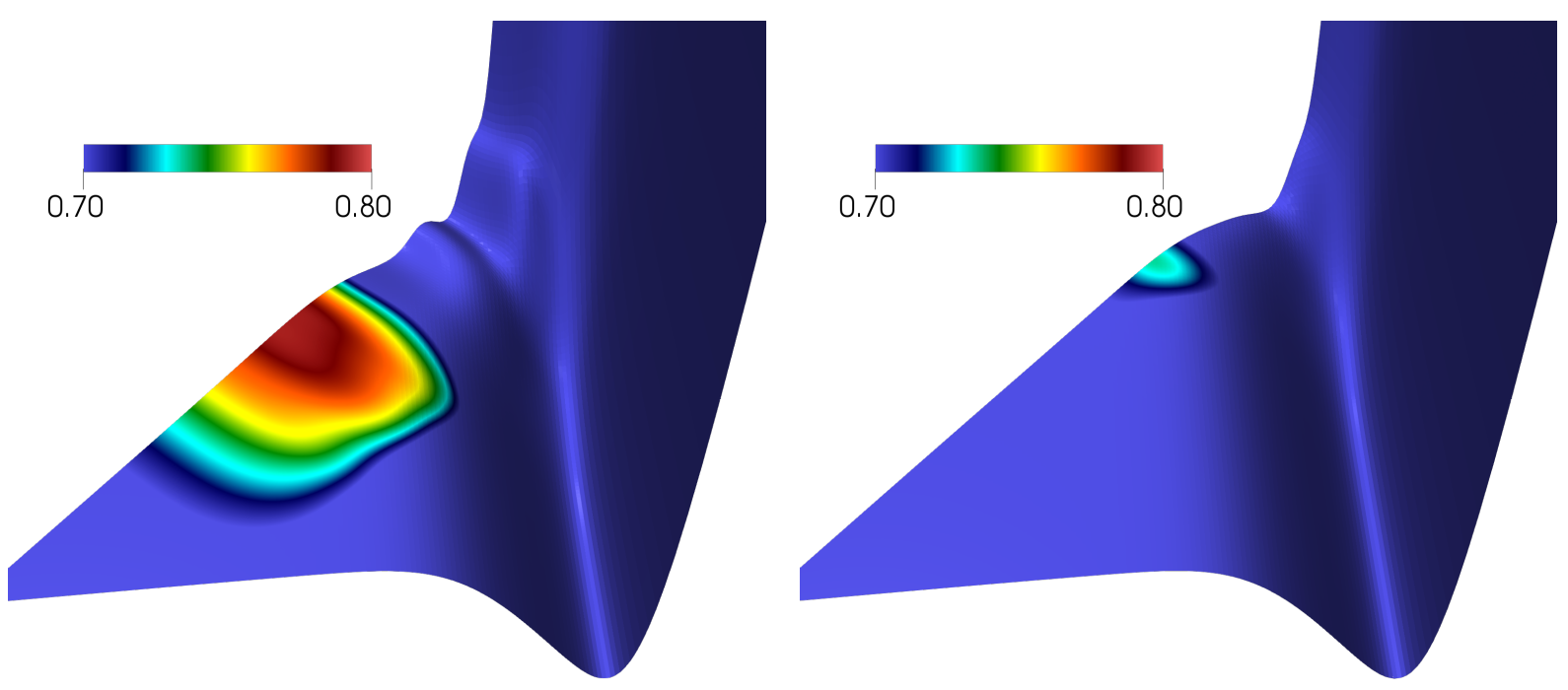

(a) $\measuredangle O_{A} O_{B} O_{C}=50^{\circ}$

(b) $\measuredangle O_{A} O_{B} O_{C}=110^{\circ}$

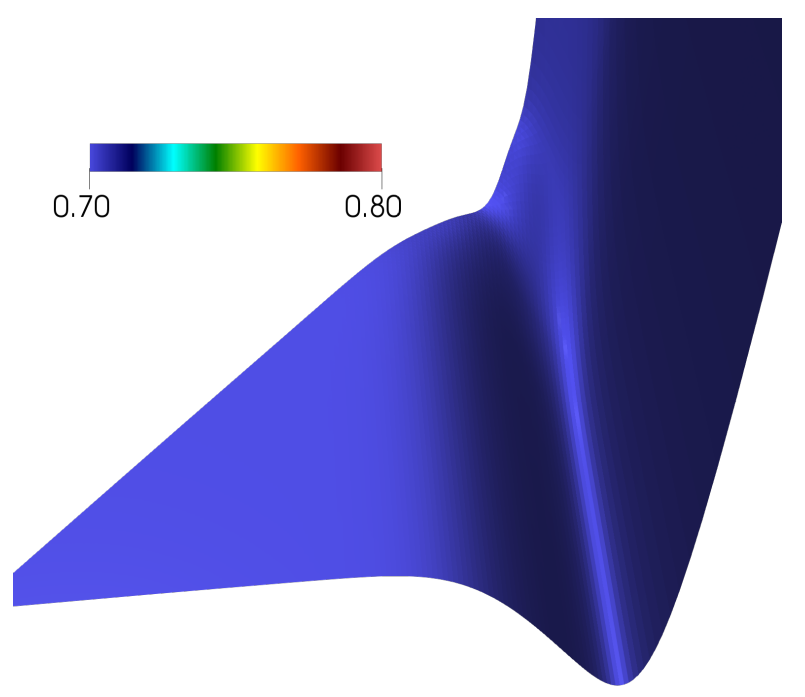

(c) $\measuredangle O_{A} O_{B} O_{C}=175^{\circ}$

FIG. S32: $3 \mathrm{D}$ views of the $2^{5} A^{\prime}$ PES resulting from the average of the 50 PIP-BNN samples for $r_{1}>r_{3}$. The surfaces have been colored based on the absolute values of Pearson correlation coefficients between the PES and the equilibrium global dissociation rate at $T_{\text {Tran }}=10000 \mathrm{~K}$. The shades of blue in the PES depth are results of rendering effects and do not correspond to different values of Pearson coefficients. 


\section{Global Dissociation Rates at QSS}
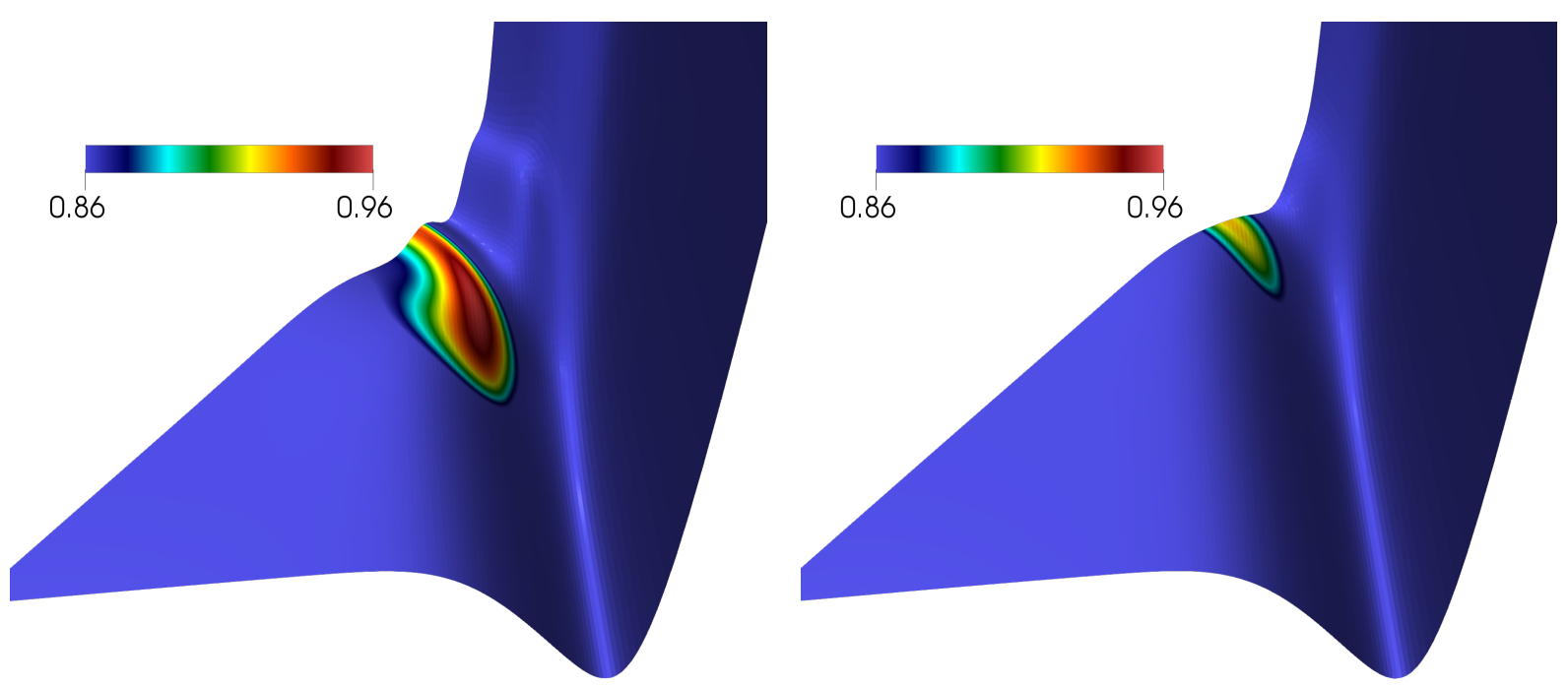

(a) $\measuredangle O_{A} O_{B} O_{C}=50^{\circ}$

(b) $\measuredangle O_{A} O_{B} O_{C}=110^{\circ}$

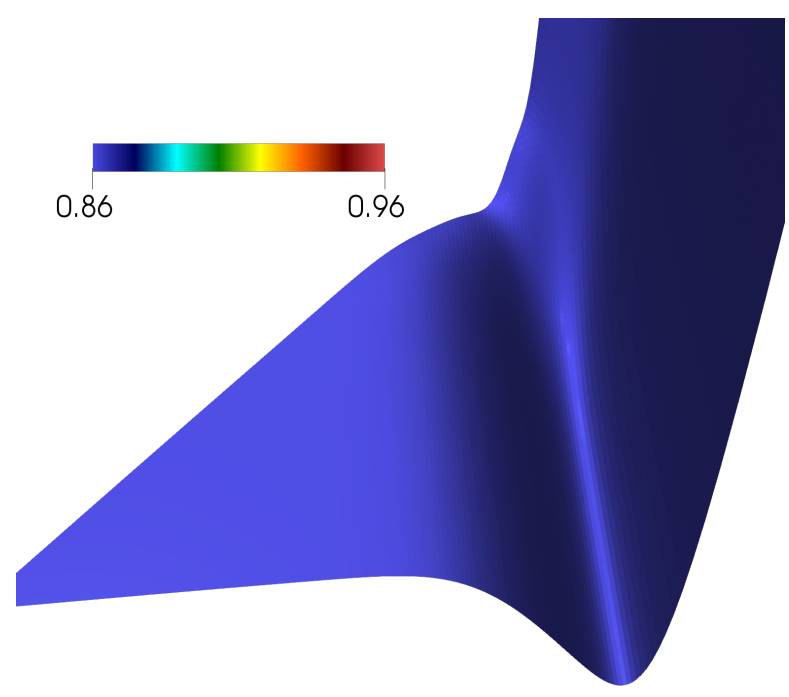

(c) $\measuredangle O_{A} O_{B} O_{C}=175^{\circ}$

FIG. S33: $3 \mathrm{D}$ views of the $2^{5} A^{\prime}$ PES resulting from the average of the 50 PIP-BNN samples for $r_{1}>r_{3}$. The surfaces have been colored based on the absolute values of Pearson correlation coefficients between the PES and the QSS global dissociation rate at $T_{\text {Tran }}=10000 \mathrm{~K}$. The shades of blue in the PES depth are results of rendering effects and do not correspond to different values of Pearson coefficients. 


\section{Global Exchange Rates at Equilibrium}
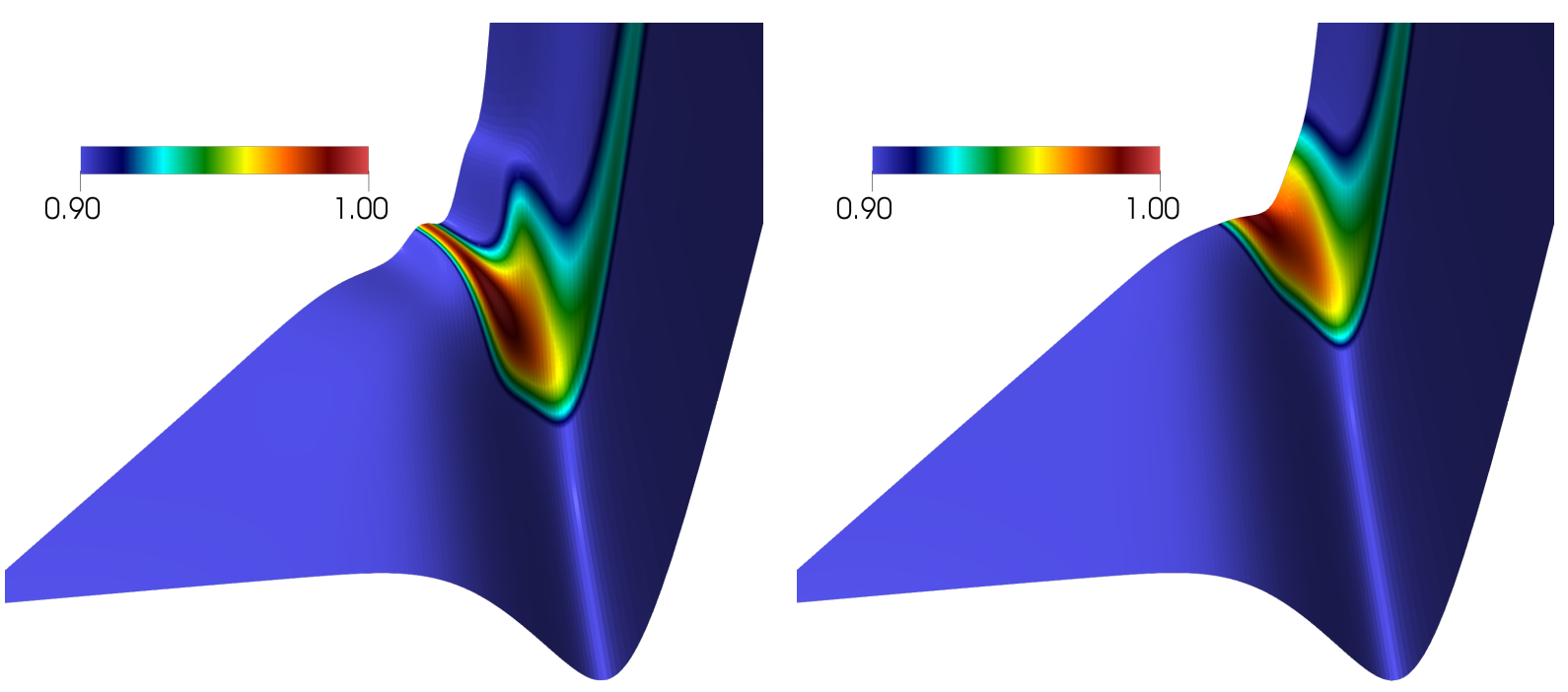

(a) $\measuredangle O_{A} O_{B} O_{C}=50^{\circ}$

(b) $\measuredangle O_{A} O_{B} O_{C}=110^{\circ}$

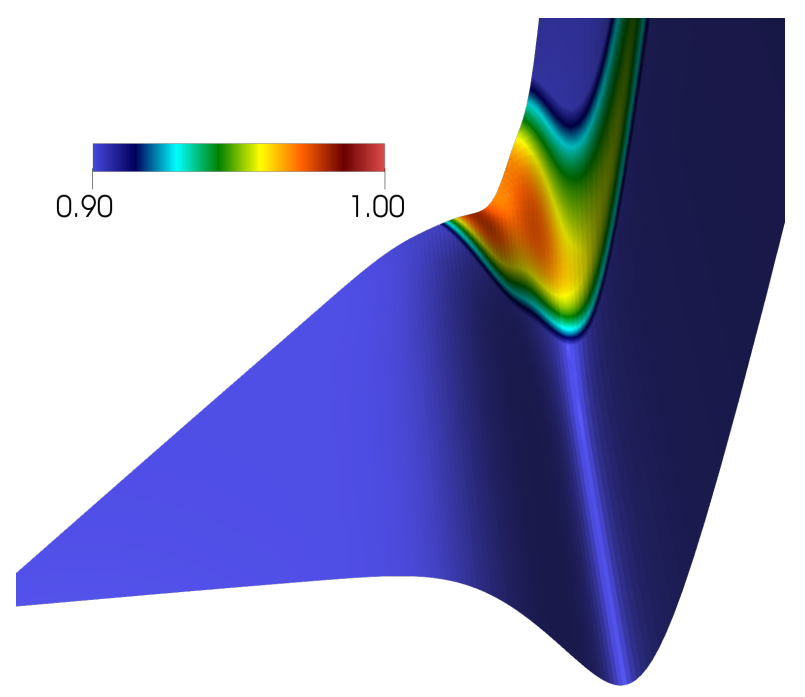

(c) $\measuredangle O_{A} O_{B} O_{C}=175^{\circ}$

FIG. S34: $3 \mathrm{D}$ views of the $2^{5} A^{\prime}$ PES resulting from the average of the 50 PIP-BNN samples for $r_{1}>r_{3}$. The surfaces have been colored based on the absolute values of Pearson correlation coefficients between the PES and the equilibrium global exchange rate at $T_{\text {Tran }}=10000 \mathrm{~K}$. The shades of blue in the PES depth are results of rendering effects and do not correspond to different values of Pearson coefficients. 


\section{Mole Fractions}
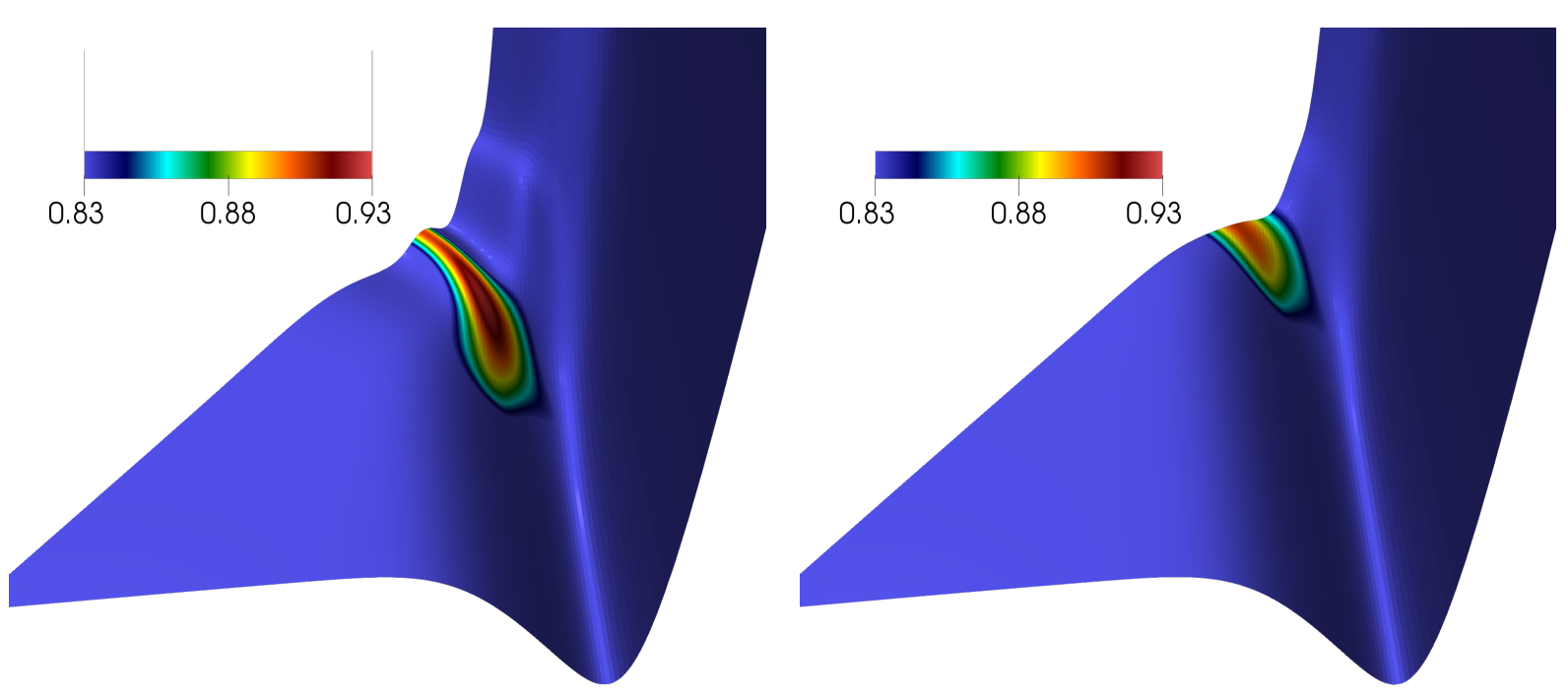

(a) $\measuredangle O_{A} O_{B} O_{C}=50^{\circ}$

(b) $\measuredangle O_{A} O_{B} O_{C}=110^{\circ}$

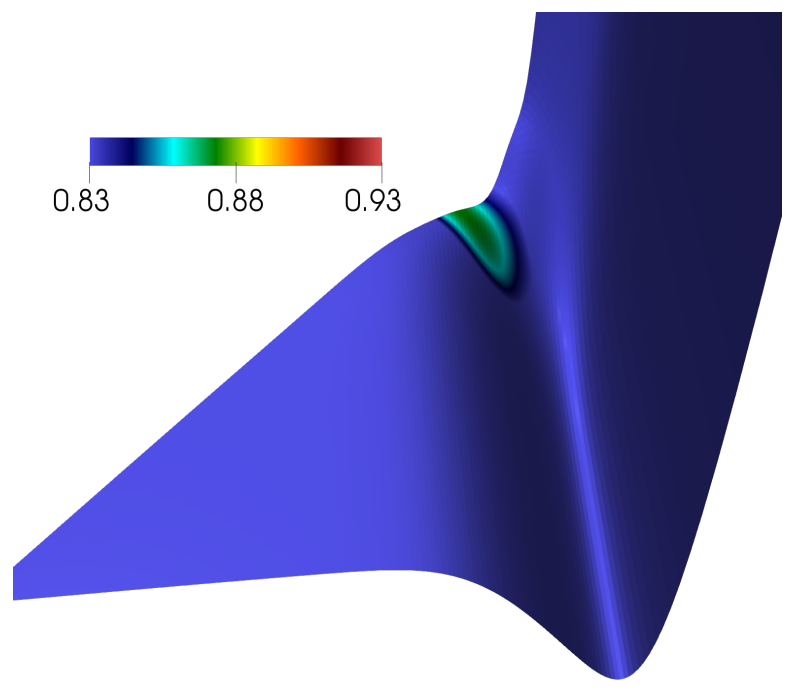

(c) $\measuredangle O_{A} O_{B} O_{C}=175^{\circ}$

FIG. S35: $3 \mathrm{D}$ views of the $2^{5} A^{\prime}$ PES resulting from the average of the 50 PIP-BNN samples for $r_{1}>r_{3}$. The surfaces have been colored based on the absolute values of Pearson correlation coefficients between the PES and the mole fraction at $T_{\text {Tran }}=10000 \mathrm{~K}$ and $\mathrm{t}=7 \mu \mathrm{s}$. The shades of blue in the PES depth are results of rendering effects and do not correspond to different values of Pearson coefficients. 